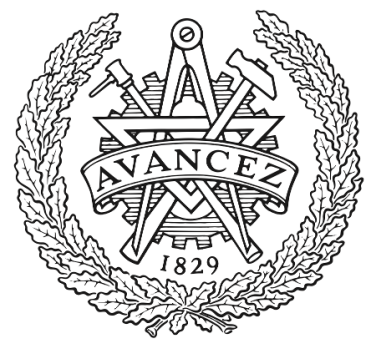

CHALMERS

UNIVERSITY OF TECHNOLOGY

\title{
Minimal excitation single-particle emitters: Comparison of charge-transport and energy-transport properties
}

Downloaded from: https://research.chalmers.se, 2023-04-26 01:15 UTC

Citation for the original published paper (version of record):

Dashti, N., Misiorny, M., Kheradsoud, S. et al (2019). Minimal excitation single-particle emitters:

Comparison of charge-transport and energy-transport properties. Physical Review B, 100(3).

http://dx.doi.org/10.1103/PhysRevB.100.035405

N.B. When citing this work, cite the original published paper. 


\title{
Minimal excitation single-particle emitters: Comparison of charge-transport and energy-transport properties
}

\author{
Nastaran Dashti, ${ }^{1}$ Maciej Misiorny, ${ }^{1}$ Sara Kheradsoud, ${ }^{2}$ Peter Samuelsson, ${ }^{2}$ and Janine Splettstoesser ${ }^{1}$ \\ ${ }^{1}$ Department of Microtechnology and Nanoscience (MC2), Chalmers University of Technology, S-412 96 Göteborg, Sweden \\ ${ }^{2}$ Department of Physics, Lund University, S-221 00 Lund, Sweden
}

(Received 5 February 2019; revised manuscript received 19 June 2019; published 3 July 2019)

\begin{abstract}
We investigate different types of time-dependently driven single-particle sources whose common feature is that they produce pulses of integer charge and minimally excite the Fermi sea. These sources are: a slowly driven mesoscopic capacitor, a Lorentzian-shaped time-dependent bias voltage, and a local gate-voltage modulation of a quantum Hall edge state. They differ by their specific driving protocols, e.g., they have a pure ac driving or a driving with a dc component. In addition, only in the first of these setups, strong confinement leading to a discrete energy spectrum of the conductor, is exploited for the single-particle emission. Here, we study if and how these basic differences impact transport properties. Specifically, we address time- and energy-resolved charge and energy currents, as well as their zero-frequency correlators (charge, energy, and mixed noise), as they are frequently used to characterize experiments in quantum optics with electrons. Beyond disparities due to a different number and polarity of particles emitted per period, we in particular identify differences in the impact, which temperature has on the observables for sources with and without energy-dependent scattering properties. We are able to characterize and quantify the effect of temperature by a small set of physically relevant parameter ratios.
\end{abstract}

DOI: 10.1103/PhysRevB.100.035405

\section{INTRODUCTION}

In recent years, transport properties, such as charge currents and their correlations have been employed as analysis tools in quantum optics with electrons [1,2]. In this field, single electrons are injected into a conductor by timedependently operated single-particle sources [3-10] and sent on quantum point contacts (QPCs) acting as beam splitters, possibly even employing quantum Hall edge states as electronic waveguides. This combination of tuneable device elements allows for implementation of optics-like experiments for electrons and has triggered a great number of further proposals, like the Mach-Zehnder [11-15], the Hanbury Brown-Twiss [16-20] or the Hong-Ou-Mandel interferometer [21-28], with additional complexities arising, for example, from the presence of the Fermi sea or from Coulomb interactions between electrons [18,25-27,29,30]. The scope of the proposed or already conducted experiments ranges from the signal analysis of the single-particle source $[16,19,20,28,31,32]$ to solid-state based entanglement protocols [15,33-37]. In addition to the study of charge currents, also energy currents $[14,38]$ and their correlations have recently come into focus [39-46], since they possibly yield further information about the spectral properties of the setup of interest.

The single-particle sources, which are used to emit electrons (and holes) into an electronic optics setup can be of very different nature [3-10]. An important requirement for the precision of these sources is that they are noiselessnamely, that the zero-frequency noise of the emitted current equals zero-meaning that they emit a regular and error-free stream of single-particle excitations. Here, we focus on those
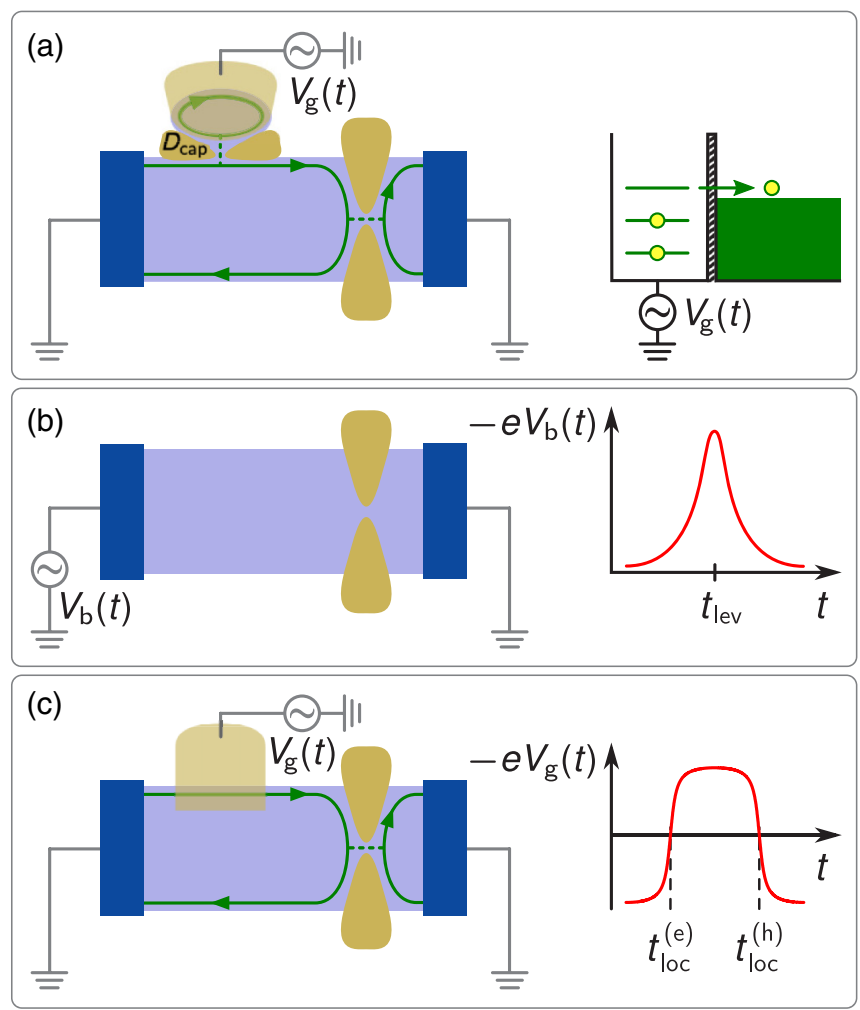

FIG. 1. Emission schemes of single particles: (A) a driven mesoscopic capacitor (cap) in a quantum-Hall $(\mathrm{QH})$ setting, with arrowed lines indicating a single conducting edge channel; (B) a voltagebiased conductor; and (C) a $\mathrm{QH}$ edge state, locally modulated by a gate potential. Currents and their correlations are detected at contact $\mathrm{R}$. 
sources, which can in addition be designed to emit these single particles as minimal excitations of the Fermi sea. More than ten years ago, a pure ac single-particle source based on a driven mesoscopic capacitor $[47,48]$ in the quantum Hall regime was first implemented [4], and it has since then been used in a variety of experimental setups (see setup A in Fig. 1). On the other hand, a very different design of a single-electron source has been realized [7,31] by shaping the time-dependent bias voltage across a conductor in a specifically chosen Lorentzian form [49-53] (see setup B in Fig. 1). Finally, a third setup was recently suggested [54], in which the local gate modulation of an edge state allows for noiseless single-particle emission on top of the Fermi sea (see setup C in Fig. 1). This third setup has not been experimentally implemented as a single-particle source yet and also its transport properties (in particular, heat transport) have been theoretically considered much less until now. While all these three types of sources enable a noiseless emission of single particles, their operation principles and specific designs differ largely. ${ }^{1}$ In experiments, which can possibly involve several synchronized sources among other device elements, it is hence of vital importance to understand, how the basic source properties reflect in the various relevant observables and when results are expected to be source-independent.

In this paper, we provide a systematic and comprehensive comparison of these three types of sources in terms of their transport characteristics. More specifically, we analyze how the properties of the different sources, schematically depicted in Fig. 1, impact the time-resolved charge and energy currents as well as the spectral current. Furthermore, inclusion of a QPC into the setup, where the initially noiseless stream of emitted particles gets scattered, allows us to study the zero-frequency charge-current and energy-current noise of the injected signals, as well as the mixed noise between charge and energy currents. For this purpose, we employ a scattering matrix approach, which is valid as long as the effective Coulomb interaction between electrons is weak. This is a reasonable assumption, since the Coulomb interaction in the only confined studied element (the mesoscopic capacitor) is typically screened by top gates. Interaction effects arising during the propagation along edge states [18,25-27,29,30] are not addressed here; we instead focus on the properties of the sources alone in order to disentangle them from other occurring effects.

We find that the zero-temperature limit of currents and noises are to a large extent similar for the three emission schemes under consideration, when operated in a regime of well-separated single-particle pulses, except for the number and type of emitted particles (electrons and holes). Most importantly, we find that the situation changes at finite temperatures. In our manuscript, we extensively study the temperature dependence of all above-mentioned transport properties. Out of the whole range of parameters describing the different sources, we identify a small, physically relevant subset of parameters, which fully characterizes this temperature dependence. Notably, we can distinguish two types of behavior,

\footnotetext{
${ }^{1}$ Other single-particle sources not addressed here inject particles far above the Fermi sea.
}

depending on whether the scattering matrix describing the single-particle source is energy-dependent or not.

The paper is organized as follows. In Sec. II, we introduce the three different emission protocols and define relevant parameter regimes for their operation. Next, the time-resolved charge and energy currents are analyzed in Sec. III, followed by a study of the spectral current in Sec. IV. The timeand energy-resolved currents are two different marginals of a Wigner function as shown in Appendix B; the full study of the Wigner function will be postponed to a future work. Finally, the zero-frequency noise of charge and energy currents, as well as their mixed noise, are discussed in Sec. V. Extensive Appendices A-F contain general definitions, full analytical results, and crucial elements of their rigorous derivations.

\section{SINGLE-PARTICLE EMISSION SCHEMES}

In this section, we introduce the three different singleparticle emission schemes that we are going to compare: a gate-voltage driven mesoscopic capacitor embedded in a quantum-Hall setup (setup A), a coherent conductor with a time-dependent Lorentzian-shaped voltage-bias driving (setup B), and a quantum Hall (QH) edge state locally modulated by a gate voltage with a smooth-box shape (setup C).

A schematic illustration of these three schemes is presented in Fig. 1. In the operation regimes, which we consider here, the common property of these three sources is that they emit a noiseless stream of single particles, as a minimal excitation just on top of the Fermi sea. In all three cases, single-particle excitations are sent from the left side onto a QPC with a single open channel with transmission $D$, which we assume to be energy-independent. The conductors are in contact with two reservoirs L (left) and R (right). The occupation of electronic states in the two reservoirs is characterized by Fermi functions $f_{\alpha}(E)=\left\{1+\exp \left[\left(E-\mu_{\alpha}\right) /\left(k_{\mathrm{B}} T\right)\right]\right\}^{-1}$, with electrochemical potentials $\mu_{\alpha},{ }^{2}$ temperature $T$, and the Boltzmann constant $k_{\mathrm{B}}$. We fix the electrochemical potential of the right reservoir as the energy reference $\mu_{\mathrm{R}}=0$. This is useful since we assume that charge and energy currents, as well as their correlations, are always detected in contact $\mathrm{R}$. In order to actually evaluate charge and energy currents, as well as their noise, we use a Floquet scattering matrix approach, see, e.g., Ref. [55], as well as Appendix A and B for definitions of all observables in terms of scattering matrices and general results. The description of the three time-dependently driven setups in terms of scattering matrices or excitation amplitudes is discussed in the following.

\section{A. Time-dependently driven mesoscopic capacitor}

The conductor of setup A is in the quantum Hall regime, so that transport takes place in chiral edge states-marked in Fig. 1 as dark green lines with arrows indicating the propagation direction. Setup A contains a mesoscopic capacitor (designated with "cap") operating as a single-particle

\footnotetext{
${ }^{2}$ In the case of time-dependent driving of the electrochemical potential (setup B), only the constant part of $\mu_{\alpha}$ enters the Fermi function, see also the Appendix of Ref. [40].
} 
source, which consists of a small region, weakly coupled to the edge by an additional QPC with transmission probability $D_{\text {cap }}$. There is no voltage bias applied, $\mu_{\mathrm{L}}=\mu_{\mathrm{R}} \equiv 0$. The capacitor region is driven at frequency $\Omega$ by a time-dependent potential $U_{\text {cap }}(t)=\bar{U}_{\text {cap }}-\delta U_{\text {cap }} \sin (\Omega t)$ induced by a top gate. Determining the relation between the relevant internal potential $U_{\text {cap }}(t)$ and the applied gate voltage $V_{\mathrm{g}}(t)$ can be highly nontrivial in this type of systems; however, a specific expression is not of direct relevance here. We hence, for simplicity, suppose the relevant internal potential $U_{\text {cap }}(t)$ to be known. The effect of this time-dependent potential is that charge is emitted into the chiral edge state and impinges onto the central QPC. The time-dependently driven mesoscopic capacitor is described by a Floquet scattering matrix $S\left(E_{n}, E\right)$, which depends on the energy $E$ of incoming states as well as on the transferred energy $n \hbar \Omega=E_{n}-E$, see, e.g., Ref. [55]. Due to the finite size of the confined capacitor region, its spectrum becomes dependent on energy. In the case we consider here, $D_{\text {cap }} \ll 1$, the capacitor spectrum is even quasi-discrete, namely, with broadened peaks at specific energies. It is the emergence of these discrete energy levels in combination with time-dependent driving, which allows to emit single particles in a controlled way. Technically, this leads to a strongly energy-dependent scattering matrix, which is an important ingredient determining the temperature-dependence of the transport properties studied in the following. Such a setup, albeit with a different (that is, square-shaped) driving potential, was first realized by Fève et al. [4]. In that case, particles are emitted at energies that, depending on the amplitude of the signal, are possibly far above the Fermi energy. In contrast, with the sinusoidal driving chosen here, we take one of the many possible driving signals, which lead to a "slow" crossing of the capacitor's discrete energy levels with the Fermi energy. This choice is appropriate for our regime of interest, where the mesoscopic capacitor emits well-separated pulses of singleparticle (electron- and hole-) excitations close to the Fermi energy. This happens under the following three conditions. First of all, (i) the driving needs to be sufficiently slow, which essentially means that the adiabatic-response condition,

$$
\frac{\Omega \tau}{D_{\text {cap }}} \ll 1,
$$

needs to be fulfilled. Here, $\tau$ stands for the time an electron needs to make one turn around the capacitor cavity. Furthermore, (ii) the condition that current pulses are well-separated in time is expressed as

$$
\Omega \sigma_{\text {cap }} \ll 1,
$$

with the temporal width of the pulses (at zero temperature) given by $\sigma_{\text {cap }}$. Thus this width needs to be much smaller than the period of the driving $\mathcal{T}=2 \pi / \Omega$. For the same reason, (iii) it needs to be guaranteed that

$$
k_{\mathrm{B}} T \ll\left|e \delta U_{\text {cap }}\right|,
$$

in other words, that the excess energy provided by a finite temperature of the contacts does not exceed the driving amplitude. However, pulses could still overlap under these last two conditions, if the constant part of the gate potential is chosen in an unfavorable way, thereby hindering the energy level of the capacitor from fully crossing the Fermi energy of the contacts during the driving cycle. For simplicity, we hence set $\bar{U}_{\text {cap }} \equiv 0$. Note that for very large driving amplitudes, that is, larger than the level spacing of the capacitor $\left|e \delta U_{\text {cap }}\right|>\Delta$, more than one electron and more than one hole can be emitted, see, e.g., Ref. [56]. Here, we avoid this situation by choosing $\left|e \delta U_{\text {cap }}\right|<\Delta$ and concentrate on the case of single-electron emission. ${ }^{3}$

Under these conditions, the energy-dependent scattering matrix takes the form

$$
S_{\text {cap }}\left(E_{n}, E\right)= \begin{cases}-2 \Omega \sigma_{\text {cap }} e^{-n \Omega \sigma_{\text {cap }}} e^{i n \Omega t_{\text {cap }}^{(\mathrm{e})}(E)} & n>0 \\ 1 & n=0 \\ -2 \Omega \sigma_{\text {cap }} e^{n \Omega \sigma_{\text {cap }}} e^{i n \Omega t_{\text {cap }}^{(\mathrm{h})}(E)} & n<0\end{cases}
$$

Here, the width of the pulses $\sigma_{\text {cap }}^{(\mathrm{e} / \mathrm{h})}$ and the energy-dependent emission times $t_{\text {cap }}^{(\mathrm{e} / \mathrm{h})}(E)$, for electrons (e) and holes (h), are related to the characteristics of the capacitor design and the driving potential as

$$
\begin{gathered}
t_{\text {cap }}^{(\mathrm{e})}(E)=\frac{E}{e \delta U_{\text {cap }} \Omega}=\frac{(2 m+1) \pi}{\Omega}-t_{\text {cap }}^{(\mathrm{h})}(E), \\
\sigma_{\text {cap }} \equiv \sigma_{\text {cap }}^{(\mathrm{h})}=\frac{D_{\text {cap }} \hbar}{2 e \delta U_{\text {cap }} \tau \Omega}=-\sigma_{\text {cap }}^{(\mathrm{e})},
\end{gathered}
$$

with $m \in \mathbb{Z}$. Here, $m$ is only required to account for emission in different driving periods; in what follows, we focus on one emission period only and fix the order in which electrons and holes are emitted, such that we can take $m=0$. Equations (5) and (6) agree with the limit for small $E /\left|e \delta U_{\text {cap }}\right|$-relevant if the condition (iii) as given in Eq. (3) is fulfilled-of the full expressions for emission times and pulse widths obtained in Ref. [57].

For the analysis below it is helpful to write the scattering matrix in a mixed time-energy representation, $S(t, E)$, related to the previously introduced Floquet scattering matrix $S\left(E_{n}, E\right)$,

$$
S_{\text {cap }}\left(E_{n}, E\right)=\int_{0}^{\mathcal{T}} \frac{d t}{\mathcal{T}} S(t, E) e^{i n \Omega t}
$$

Then, the resonant emission of electrons and holes in the vicinity of the emission times $t_{\text {cap }}^{(j)}$ becomes particularly clear

$$
S_{\text {cap }}(t, E)=\sum_{j=\mathrm{e}, \mathrm{h}} \frac{t-t_{\mathrm{cap}}^{(j)}(E)-i \sigma_{\mathrm{cap}}^{(j)}}{t-t_{\mathrm{cap}}^{(j)}(E)+i \sigma_{\mathrm{cap}}^{(j)}} .
$$

Equivalently, one can rewrite the scattering matrix above in terms of relevant emission energies. This formulation emphasizes that an energy level of the discrete spectrum of the capacitor with width $\Gamma$ has to be energetically accessible in order to allow for the emission of electrons and holes,

$$
S_{\text {cap }}(t, E)=\sum_{j=\mathrm{e}, \mathrm{h}} \frac{E-E_{\mathrm{res}}^{(j)}(t)-i \Gamma}{E-E_{\mathrm{res}}^{(j)}(t)+i \Gamma},
$$

\footnotetext{
${ }^{3}$ Note that $\Delta$ does not directly enter our model: the condition $\left|e \delta U_{\text {cap }}\right|<\Delta$ allows us to consider a single-level spectrum for the capacitor.
} 
with the level broadening $\Gamma$ and the emission energies $E_{\mathrm{res}}^{(j)}(t)$ defined as

$$
\begin{gathered}
E_{\mathrm{res}}^{(\mathrm{e})}(t)=e \delta U_{\text {cap }} \Omega t=e \delta U_{\text {cap }} \pi-E_{\mathrm{res}}^{(\mathrm{h})}(t), \\
\Gamma=\frac{\hbar D_{\text {cap }}}{2 \tau}=e \delta U_{\text {cap }} \Omega \sigma_{\text {cap }} .
\end{gathered}
$$

The adiabatic condition (i) and the resonance condition (ii), given in Eqs. (1) and (2), respectively, can then be alternatively formulated in terms of energy scales only,

$$
\hbar \Omega \ll 2 \Gamma \text { and } \hbar \Omega \ll 2 \mathcal{E} .
$$

Here, we have introduced the energy,

$$
\mathcal{E}=\hbar /\left(2 \sigma_{\text {cap }}\right),
$$

which will later turn out to characterize the energy carried by an emitted pulse. Note that while the energy carried by the pulse $\mathcal{E}$ is a relevant concept for the single-particle emission from all three setups, the level width $\Gamma$ is a parameter that only describes the mesoscopic capacitor with its discrete spectrum arising due to the confinement. In particular, $\Gamma$ is independent of the driving.

\section{B. Lorentzian bias voltage}

The conductor of setup B does not require a magnetic field. In order to produce controlled single-particle excitations in setup $\mathrm{B}$, a time-dependent potential is applied to the left contact $\mu_{\mathrm{L}}(t) /(-e) \equiv V_{\mathrm{b}}(t)=\delta V_{\mathrm{b}}(t)+\bar{V}_{\mathrm{b}}$. Here, $\delta V_{\mathrm{b}}(t)$ and $\bar{V}_{\mathrm{b}}$ stand for the pure ac and dc components of the driving potential $V_{\mathrm{b}}(t)$, respectively, and $-e$ (with $e>0$ ) is the electron charge. The application of a time-dependent bias voltage $V_{\mathrm{b}}(t)$ leads to a spread in energy of the electronic states injected from the left contact and impinging on the scatterer [47]. Therefore one has to relate the creation and annihilation operators $\hat{a}_{\mathrm{L}}^{\dagger}(E)$ and $\hat{a}_{\mathrm{L}}(E)$, for current-carrying states entering the conductor after having been subject to the time-dependent driving, to those deep in the contact, referred to as $\hat{a}_{\mathrm{L}}^{0 \dagger}(E)$ and $\hat{a}_{\mathrm{L}}^{0}(E)$. Importantly, only the latter operators obey Fermi-Dirac statistics, $\left\langle\hat{a}_{\mathrm{L}}^{0 \dagger}(E) \hat{a}_{\mathrm{L}}^{0}\left(E^{\prime}\right)\right\rangle=\delta\left(E-E^{\prime}\right) f_{\mathrm{L}}(E)$. The relation between the operators $\hat{a}_{\mathrm{L}}(E)$ and $\hat{a}_{\mathrm{L}}^{0}(E)$ is

$$
\hat{a}_{\mathrm{L}}(E)=\sum_{n=-\infty}^{\infty} c_{\mathrm{L}, n} \hat{a}_{\mathrm{L}}^{0}\left(E_{-n}\right),
$$

with the amplitude $c_{\mathrm{L}, n}$,

$$
c_{\mathrm{L}, n}=\int_{0}^{\mathcal{T}} \frac{d t}{\mathcal{T}} e^{i n \Omega t} e^{-i \varphi_{\mathrm{L}}(t)},
$$

basically defined as the Fourier coefficient of the phase factor $\exp \left[-i \varphi_{\mathrm{L}}(t)\right]$, and the time-dependent phase being

$$
\varphi_{\mathrm{L}}(t)=-\frac{e}{\hbar} \int_{0}^{t} d t^{\prime} \delta V_{\mathrm{b}}\left(t^{\prime}\right) .
$$

The amplitudes $c_{\mathrm{L}, n}$ determine the probability $\left|c_{\mathrm{L}, n}\right|^{2}$ that $n$ Floquet energy quanta $\hbar \Omega$ are emitted $(n<0)$ or absorbed $(n>0)$ in a scattering process. Note that in contrast to the scattering matrix of setup A, these amplitudes given by Eq. (15) are energy-independent.
Here, we are interested in a specific shape of the driving signal, namely, a periodically repeated Lorentzian-shaped bias,

$$
V_{\mathrm{b}}(t)=V \mathcal{T} \sum_{j=-\infty}^{\infty} \mathscr{L}_{\sigma_{\mathrm{lev}}}\left(t-t_{\mathrm{lev}}-j \mathcal{T}\right),
$$

with the Lorentzian function $\mathscr{L}_{y}(x)=y\left[\pi\left(x^{2}+y^{2}\right)\right]^{-1}$. The voltage pulse is centered around $t_{\mathrm{lev}}$, which we refer to as the particle emission time, and has a full width at half maximum given by $2 \sigma_{\text {lev }}$. It has been theoretically predicted [49-51], and later also verified in experiments [7,31], that under the condition $V=\hbar \Omega /(-e)$, such a Lorentzian-shaped driving signal leads to a noiseless emission of independent singleelectron excitations (one per period), known as levitons. This is the regime, which we will address in this paper, where equivalent to the condition given in Eq. (2), we require $\Omega \sigma_{\text {lev }} \ll 1$ to guarantee that pulses are well separated in time. In this resonant regime, the amplitudes $c_{\mathrm{L}, n}$ take the form

$$
c_{\mathrm{L}, n}= \begin{cases}-2 \Omega \sigma_{\mathrm{lev}} e^{-n \Omega \sigma_{\mathrm{lev}}} e^{i(n+1) \Omega \mathrm{t}_{\mathrm{lev}}} & n>-1, \\ -e^{-\Omega \sigma_{\mathrm{lev}}} & n=-1, \\ 0 & n<-1 .\end{cases}
$$

\section{Local time-dependent edge-state modulation}

Setup C combines some of the properties of the previously introduced setups A and B, see Fig. 1. Similar as in the mesoscopic capacitor setup, the conductor is in the quantum Hall regime and locally driven by applying a pure ac gate voltage. However, no confinement is exploited here, insteadsimilar as in setup B-it is the specifically chosen shape of the driving gate voltage which leads to the noiseless emission of particles (here both electrons and holes). The scattering matrix describing the effect of the induced electric field on electrons traversing the gated region is given by

$$
\begin{aligned}
S_{\mathrm{loc}}(t, E) & =e^{i E \tau_{\mathrm{g}} / \hbar} e^{-i \varphi_{\mathrm{g}}(t)} \\
& =e^{i E \tau_{\mathrm{g}} / \hbar} \sum_{n=-\infty}^{\infty} c_{\mathrm{g}, n} e^{-i n \Omega t} .
\end{aligned}
$$

Here, $\tau_{\mathrm{g}}$ denotes the traversal time of an electron passing through the gated region. The amplitudes $c_{\mathrm{g}, n}$ are defined analogously to Eq. (15), with

$$
\varphi_{\mathrm{g}}(t)=-\frac{e}{\hbar} \int_{t-\tau_{\mathrm{g}}}^{t} d t^{\prime} \delta U_{\mathrm{loc}}\left(t^{\prime}\right) .
$$

The relation between the internal potential $\delta U_{\mathrm{loc}}\left(t^{\prime}\right)$ in the interacting region and the potential $\delta V_{\mathrm{g}}(t)$ applied to the gate can, as mentioned before, in general be fairly nontrivial [54]. Here, for simplicity, we focus on the adiabaticresponse regime, requiring $\tau_{R C} \ll \mathcal{T}$. The $R C$-time, $\tau_{R C}=$ $R C_{\mu}$, is the product of the Büttiker resistance $[47,48] R=$ $h /\left(2 e^{2}\right)$, first experimentally demonstrated by Gabelli et al. [58] and the total (electrochemical) capacitance $C_{\mu}$, which for a metallic gate is given by $C_{\mu}^{-1}=C^{-1}+C_{\mathrm{q}}^{-1}$, with the purely electrostatic capacitance $C$ and the quantum capacitance $C_{\mathrm{q}}=\tau_{\mathrm{g}} e^{2} / h$. In the adiabatic-response limit, one derives 


$$
\begin{aligned}
& \delta U_{\mathrm{loc}}(t)=\left(C_{\mu} / C_{\mathrm{q}}\right) \delta V_{\mathrm{g}}(t) \text { and } \\
& \qquad \varphi_{\mathrm{g}}(t)=2 \pi \frac{C_{\mu}}{(-e)} \delta V_{\mathrm{g}}(t) .
\end{aligned}
$$

In order to obtain noiseless emission of electrons and holes in this driving regime, the required ac gate potential is a smoothbox potential

$$
\delta V_{\mathrm{g}}(t)=\frac{\delta V_{\mathrm{g}}}{2 \pi} \operatorname{Re}\left\{i \ln \left[\frac{\sin \left(\Omega\left[t-t_{\mathrm{loc}}^{(\mathrm{e})}+i \sigma_{\mathrm{loc}}\right] / 2\right)}{\sin \left(\Omega\left[t-t_{\mathrm{loc}}^{(\mathrm{h})}+i \sigma_{\mathrm{loc}}\right] / 2\right)}\right]\right\}-\frac{\delta V_{\mathrm{g}}}{2},
$$

fulfilling the requirement $C_{\mu} \delta V_{\mathrm{g}} /(-e)=1$. Here, we assume that the steps of the box are evenly distributed, i.e., $t_{\mathrm{loc}}^{(\mathrm{h})}-$ $t_{\text {loc }}^{(\mathrm{e})}=\mathcal{T} / 2$, meaning that the emission time of electrons and holes is half a period apart. Moreover, these pulses are well separated in time if $\Omega \sigma_{\text {loc }} \ll 1$. Then, the coefficients $c_{\mathrm{g}, n}$ in Eq. (19) read

$$
c_{\mathrm{g}, n}= \begin{cases}-2 \Omega \sigma_{\mathrm{loc}} e^{-n \Omega \sigma_{\mathrm{loc}}} e^{i n \Omega t_{\mathrm{loc}}^{(\mathrm{e})}} & n>0 \\ 1 & n=0 \\ -2 \Omega \sigma_{\mathrm{loc}} e^{n \Omega \sigma_{\mathrm{loc}}} e^{i n \Omega t_{\mathrm{loc}}^{(\mathrm{h})}} & n<0\end{cases}
$$

\section{Common and distinct features}

While all of the three introduced types of sources lead to the emission of a regular stream of particles, which only minimally excites the Fermi sea, they differ in their specific setup and operation. Therefore, in a concrete experiment, one type of single-particle source can have advantages or disadvantages with respect to another. For example, setups A and $\mathrm{C}$ involve edge states, which can be used as electronic waveguides in quantum optics setups for electrons, but the particle propagation along those edge states is also known to imply complicated interaction-induced decoherence effects. Setups B and C are very simple in their design, but require careful tuning of the signal shape.

In what follows, we are going to discuss the currents of these three different types of sources and the related noise in different regimes, within the approximations fixed above. In order to compare the properties of the currents from the three sources, we take them to be characterized by equal widths and emission times

$$
\begin{gathered}
\sigma \equiv \sigma_{\text {cap }}=\sigma_{\mathrm{lev}}=\sigma_{\mathrm{loc}}, \\
t^{(\mathrm{e})} \equiv t_{\mathrm{lev}}=t_{\mathrm{cap}}^{(\mathrm{e})}(E=0)=t_{\mathrm{loc}}^{(\mathrm{e})}=0,
\end{gathered}
$$

where we focus on one emission period and only display electronic emission times.

In our comparison, we are particularly interested in the influence of temperature [59-61]; we will demonstrate that the different dependence of observables on temperature might constitute a further reason to prefer one type of source instead of the other. To make this comparison transparent, we define two dimensionless parameters

$$
\begin{aligned}
\alpha_{\mathcal{E}} & :=\frac{k_{\mathrm{B}} T}{\mathcal{E}}, \\
\alpha_{\Gamma} & :=\frac{k_{\mathrm{B}} T}{\Gamma},
\end{aligned}
$$

which are not fixed by the described conditions of adiabaticity and emission of separate pulses. They define the ratio of temperature with respect to the energy carried by the emitted pulses $\left(\alpha_{\mathcal{E}}\right)$, as well as to the driving-independent level width of the capacitor's energy spectrum $\left(\alpha_{\Gamma}\right)$. It turns out that these two parameters fully determine the temperature dependence of the considered observables in the three single-particle sources. Identifying furthermore the width of the pulses as relevant timescale for the physics of the devices, and the energy emitted per pulse as the relevant energy scale, we introduce the dimensionless time and energy variables

$$
\begin{gathered}
\tilde{t}=t / \sigma, \\
\widetilde{E}=E / \mathcal{E} .
\end{gathered}
$$

Those four variables, Eqs. (26)-(29), will be used to make our analytical and numerical findings more compact and insightful.

\section{TIME-RESOLVED CURRENTS}

In order to understand how these relevant energy scales enter the time-resolved charge and energy currents emitted from the sources, it is helpful to consider the general expression for currents in the right contact

$$
\begin{aligned}
\left(\begin{array}{c}
I(t) \\
I^{\mathcal{E}}(t)
\end{array}\right)= & \frac{D}{h} \sum_{n, \ell=-\infty}^{\infty} \int d E\left(\begin{array}{c}
-e \\
E+\frac{(n+\ell) \hbar \Omega}{2}
\end{array}\right) \\
& \times\left[f_{\mathrm{L}}(E)-f_{\mathrm{R}}\left(E_{n}\right)\right] \int_{0}^{\mathcal{T}} \frac{d t^{\prime}}{\mathcal{T}} e^{i n \Omega\left(t-t^{\prime}\right)} \\
& \times \int_{0}^{\mathcal{T}} \frac{d t^{\prime \prime}}{\mathcal{T}} e^{-i \ell \Omega\left(t-t^{\prime \prime}\right)} S^{*}\left(t^{\prime}, E\right) S\left(t^{\prime \prime}, E\right) ;
\end{aligned}
$$

see also Appendix B. For the driven mesoscopic capacitor (setup A) and the locally modulated edge state (setup C), the subscript of the Fermi functions can be dropped. Moreover, in order to describe the leviton emission (setup B), scattering matrices have to be replaced by $S(t, E) \rightarrow \exp \left[-i \varphi_{\mathrm{L}}(t)\right]$.

First of all, it can be noticed that in Eq. (30), there is the energy dependence given by the scattering matrix itself. This energy dependence is always absent for the leviton emission and also for the locally modulated edge state the energy dependence of the scattering matrix does not contribute to Eq. (30). However, also for the mesoscopic capacitor-when driven slowly - the energy dependence of the scattering matrix can be neglected, as long as it does not occur on energy scales exceeding the one set by the driving. This latter is given by the parameter $\mathcal{E}$, as defined in Eq. (13). Importantly, in Eq. (30), another energy scale enters through the Fermi functions: the temperature. As soon as the energy scale given by temperature, $k_{\mathrm{B}} T$ is much bigger than the energy scale set by the driving, $\mathcal{E}$, or in other words, if the parameter $\alpha_{\mathcal{E}}=k_{\mathrm{B}} T / \mathcal{E}$ defined in Eq. (26) fulfils $\alpha_{\mathcal{E}} \gg 1$, the energy dependence of the scattering matrix of the slowly driven mesoscopic capacitor becomes relevant. Technically this implies that for the low-temperature regime with $\alpha_{\mathcal{E}}=k_{\mathrm{B}} T / \mathcal{E} \lesssim$ 1 , we can perform the replacement $S(t, E) \rightarrow S(t, 0)$, while this replacement is not correct in the high-temperature regime defined by $\alpha_{\mathcal{E}}=k_{\mathrm{B}} T / \mathcal{E} \gg 1$. 
As a result, in the limits considered here, the time-resolved currents going along with the single-particle emission from the three sources have basically equivalent features when $\alpha_{\mathcal{E}} \lesssim 1$. The only differences then stem from the fact that in one driving period one electron and one hole are emitted from the capacitor and the locally modulated edge state, while the bias-driving results in the emission of particles of one polarity, only. In what follows, we discuss features of time-resolved currents for the leviton emission and the locally modulated edge state always together with the low- to intermediatetemperature regime $\left(\alpha_{\mathcal{E}} \lesssim 1\right)$ of the mesoscopic capacitor, since the behavior is basically equivalent.

Importantly, at higher temperatures, $\alpha_{\mathcal{E}} \gg 1$, the signal emitted from the mesoscopic capacitor is modified-in contrast to the leviton and the signal emitted from the locally modulated edge state, which always keep their lowtemperature features.

\section{A. Time-resolved charge current}

\section{Low and intermediate temperatures, $\alpha_{\mathcal{E}} \lesssim 1$}

For a given period, the time-resolved charge current, see, e.g., Ref. [55], takes the form

$$
\frac{I_{\mathrm{cap} / \mathrm{loc}}(\tilde{t})}{-e / \sigma}=\frac{D}{\pi}\left\{\frac{1}{\tilde{t}^{2}+1}-\frac{1}{\left(\tilde{t}-\frac{\mathcal{T}}{2 \sigma}\right)^{2}+1}\right\},
$$

with the electron-emission followed by the hole-emission after a half period. Here, we express the time-resolved current as function of the dimensionless time $\tilde{t}=t / \sigma$, as introduced in Eq. (28). This clearly shows that the relevant timescale for the particle emission is the pulse width $\sigma$; we will hence use this compact notation also in other analytic results for time-resolved currents throughout this section. The specific shape of Eq. (31) stems from our choice for the emission times $t^{(\mathrm{e})}=0$ (corresponding to $\bar{U}=0$ for the driven capacitor). The shape of the current is Lorentzian with the width $\sigma$ for both emission schemes, see setups A and C in Fig. 1. The time-resolved current (31) is plotted as a solid red line in Fig. 2(a). The leviton emission is described by the first (electron-emission) component, only; see the black dashed line in Fig. 2(a).

\section{High temperature, $\alpha_{\mathcal{E}} \gg 1$}

When the energy scale set by temperature exceeds the energy $\mathcal{E}$ associated with the driving, the energy dependence of the scattering matrix of the mesoscopic capacitor can be resolved even in the limit of slow driving. This yields important differences in the current properties. The time-resolved charge current emitted from the driven capacitor in this limit reads

$$
\begin{aligned}
\frac{I_{\text {cap }}(t)}{-e}= & D \sum_{j=\mathrm{e}, \mathrm{h}} \frac{\partial E_{\text {res }}^{(j)}(t)}{\partial t} \\
& \times \int d E\left[-\frac{\partial f(E)}{\partial E}\right] \mathscr{L}_{\Gamma}\left(E-E_{\text {res }}^{(j)}(t)\right) .
\end{aligned}
$$

Here, we have introduced the emission energy $E_{\text {res }}^{(j)}(t)$, defined in Eq. (10). One can see that the energy dependence of the scattering matrix, which is determined by the Lorentzian
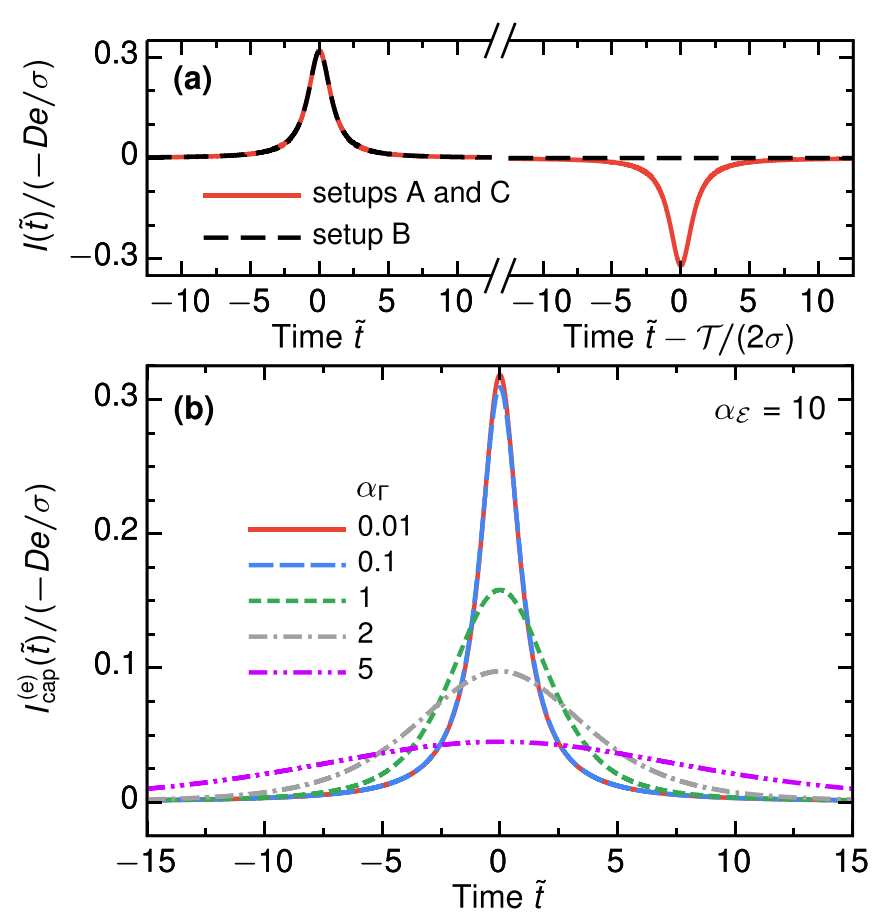

FIG. 2. Charge current as a function of the dimensionless time $\tilde{t}=t / \sigma$ for $\alpha_{\mathcal{E}}=k_{\mathrm{B}} T / \mathcal{E}=10$ and $\Omega \sigma \ll 1$. (a) shows the charge currents emitted from the driven capacitor or the locally modulated edge state (setups A and C, respectively; see solid line) and from a Lorentzian-shaped bias voltage pulse (setup B; see dashed line) for relevant times of the whole driving period. Note the cut of the axis in the center. (b) presents how the pulse emitted from the capacitor in the first half-period (i.e., the electron-emission contribution) changes with different values of $\alpha_{\Gamma}=k_{\mathrm{B}} T / \Gamma$, while the leviton pulse and the pulse emitted in setup $\mathrm{C}$ remain unaltered.

$\mathscr{L}_{\Gamma}$ with width $\Gamma$, is irrelevant as long as $\alpha_{\Gamma}=k_{\mathrm{B}} T / \Gamma \ll 1$, namely, as long as temperature is smaller than the energy-level width of the capacitor spectrum. Hence, in this limit, the result for the current is identical to the low-temperature result given in Eq. (31).

In contrast, the energy dependence of the scattering matrix becomes relevant, when the temperature reaches the level width, $\alpha_{\Gamma} \geqslant 1$, demonstrating that it is exactly this factor $\alpha_{\Gamma}$ which determines the temperature dependence of the timeresolved current. For very large temperatures, $\alpha_{\Gamma} \gg 1$, we find that the current is strongly suppressed and has a shape dictated by the derivative of the Fermi function,

$$
I_{\text {cap }}(t)=e D \sum_{j=\mathrm{e}, \mathrm{h}} \frac{\partial f\left(E_{\mathrm{res}}^{(j)}(t)\right)}{\partial t} .
$$

The role of the factor $\alpha_{\Gamma}$, can be seen best from the explicit result

$$
\frac{I_{\text {cap }}^{(\mathrm{e})}(\tilde{t})}{-e / \sigma}=\frac{D}{\alpha_{\Gamma}} \cdot \frac{e^{\tilde{t} / \alpha_{\Gamma}}}{\left[1+e^{\tilde{t} / \alpha_{\Gamma}}\right]^{2}} .
$$

Here, for simplicity, we show the electron-emission contribution only. The hole-emission contribution is given by an equivalent expression with an opposite sign. The general result, obtained from carrying out the energy integration in Eq. (32), is given in Appendix C 1. This general result is plotted in 
Fig. 2(b), where we show results for the time-resolved charge current in all temperature regimes. The suppression of the current for high temperatures, as well as the evolution of its characteristic shape from a Lorentzian to a derivative of a Fermi function become visible there.

\section{B. Time-resolved energy current}

\section{Low and intermediate temperatures, $\alpha_{\mathcal{E}} \lesssim 1$}

The time-resolved energy current at low temperatures has very similar features as the charge current, Eq. (31), where an energy $\mathcal{E}$, is transported per pulse instead of a charge $-e$ (or $e$ for holes),

$$
\frac{I_{\mathrm{cap} / \mathrm{loc}}^{\mathcal{E}}(\tilde{t})}{\mathcal{E} / \sigma}=\frac{2 D}{\pi}\left\{\frac{1}{\left[\tilde{t}^{2}+1\right]^{2}}+\frac{1}{\left[\left(\tilde{t}-\frac{\mathcal{T}}{2 \sigma}\right)^{2}+1\right]^{2}}\right\} .
$$

Note that both electron and hole pulses carry the same energy and that the pulse shape is sharper than the one of the charge current. Again, the leviton emission results in an energy current described by the first (electron-emission) contribution, only; see black dashed line in Fig. 3(a). The result for setups A and C, Eq. (35), is plotted as a solid red line in Fig. 3(a).

\section{High temperature, $\alpha_{\mathcal{E}} \gg 1$}

The energy dependence of the scattering matrix of the driven mesoscopic capacitor, which gets important in the regime $\alpha_{\mathcal{E}} \gg 1$, has a much stronger effect on the energy current than on the charge current. We find for the timeresolved energy current from the driven capacitor

$$
\begin{aligned}
I_{\text {cap }}^{\mathcal{E}}(t)= & D \sum_{j=\mathrm{e}, \mathrm{h}} \frac{\partial E_{\mathrm{res}}^{(j)}(t)}{\partial t} \int d E\left[-\frac{\partial f(E)}{\partial E}\right] \\
& \times\left\{E \mathscr{L}_{\Gamma}\left(E-E_{\mathrm{res}}^{(j)}(t)\right)\right. \\
& +\pi \hbar \frac{\partial E_{\mathrm{res}}^{(j)}(t)}{\partial t}\left[\left[\mathscr{L}_{\Gamma}\left(E-E_{\mathrm{res}}^{(j)}(t)\right)\right]^{2}\right. \\
& \left.\left.+E \frac{\partial\left[\mathscr{L}_{\Gamma}\left(E-E_{\mathrm{res}}^{(j)}(t)\right)\right]^{2}}{\partial E}\right]\right\} .
\end{aligned}
$$

Again, as long as $\alpha_{\Gamma} \ll 1$, the same expression as Eq. (35) is obtained. However, when temperature is of the order of $\Gamma$ or larger, the current gets significantly modified, see Appendix C 2 for a full analytical expression. Equation (36) consists formally of two contributions: one that is antisymmetric and the other that is symmetric around the emission time. The antisymmetric part has a shape similar to the time-resolved charge current, given in Eq. (32). It can be understood as the energy carried by each particle, and hence, it changes its sign depending on whether the particle is injected above or below the reference electrochemical potential, $\mu_{R}=0$. Obviously, this term can only considerably contribute at large temperatures, where states both above and below the electrochemical potential are available for the single-particle emission. The second, symmetric part is the one that yields the low-temperature result for $\alpha_{\Gamma} \ll 1$. The latter describes the
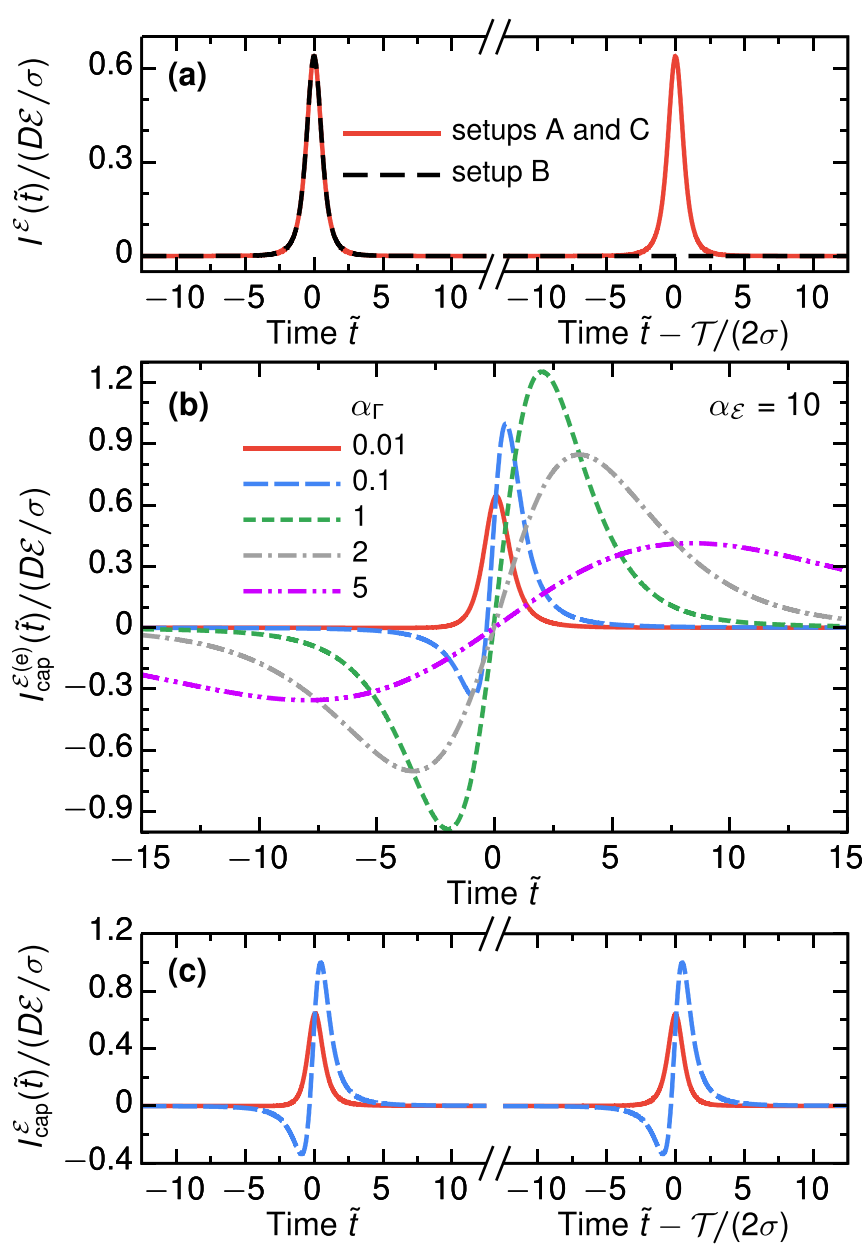

FIG. 3. Energy current as a function of the dimensionless time $\tilde{t}=t / \sigma$ for $\alpha_{\mathcal{E}}=k_{\mathrm{B}} T / \mathcal{E}=10$ and $\Omega \sigma \ll 1$. (a) and (b) are analogous to those shown in Fig. 2 except that now the energy current is presented. (c) illustrates both electron- and hole-emission contributions to the energy current emitted from the driven capacitor for two selected values of $\alpha_{\Gamma}=k_{\mathrm{B}} T / \Gamma$ plotted in (b).

energy injected into the conductor necessary for the excitation of a particle in the presence of the Fermi sea. Temperature also modifies this term, since the time at which this energy is injected into the conductor depends on the broadening of the level due to the coupling, $\Gamma$, and on temperature. See Appendix D for a more detailed discussion of the two contributing terms. The characteristics of these two contributions become particularly evident in the limit $\alpha_{\Gamma} \gg 1$, where we find the insightful form of the current

$$
\begin{aligned}
I_{\text {cap }}^{\mathcal{E}}(t)= & D \sum_{j=\mathrm{e}, \mathrm{h}} E_{\mathrm{res}}^{(j)}(t) \\
& \times\left\{-\frac{\partial f\left(E_{\mathrm{res}}^{(j)}(t)\right)}{\partial t}+\frac{\hbar}{2 \Gamma} \frac{\partial^{2} f\left(E_{\mathrm{res}}^{(j)}(t)\right)}{\partial t^{2}}\right\} .
\end{aligned}
$$

Which of these two terms dominates depends both on $\alpha_{\Gamma}$ and on $\alpha_{\mathcal{E}}$, This is a fundamental difference with respect to the time-resolved charge current, which-at high temperaturesis governed exclusively by the coefficient $\alpha_{\Gamma}$. It can be most 
clearly seen from the explicit evaluation of Eq. (37), leading to

$$
\begin{aligned}
\frac{I_{\text {cap }}^{\mathcal{E}(\mathrm{e})}(\tilde{t})}{\mathcal{E} / \sigma}= & D \frac{\left(\tilde{t} / \alpha_{\Gamma}\right) e^{\tilde{t} / \alpha_{\Gamma}}}{\left[1+e^{\tilde{t} / \alpha_{\Gamma}}\right]^{2}} \\
& \times \frac{\Gamma}{\mathcal{E}}\left[1+\frac{1}{\alpha_{\mathcal{E}}} \tanh \left(\frac{\tilde{t}}{2 \alpha_{\Gamma}}\right)\right],
\end{aligned}
$$

where for simplicity, we show only the electron-emission contribution. In Fig. 3(b), we display the time-resolved energy current for different values of $\alpha_{\Gamma}$, starting from the general expression given in Appendix C 2. The more the temperature increases with respect to the level width $\Gamma$, the more the properties of the time-resolved energy current change. The peak at the emission time, found for low temperatures, evolves into an antisymmetric curve, which vanishes in the vicinity of the emission time. The energy transferred during the emission of electrons and holes is then almost fully governed by the temperature broadening of the Fermi sea: particles injected at negative/positive energies lead to negative/positive energy currents. ${ }^{4}$ The maximum and minimum of this close-to antisymmetric curve is shifted to $t \approx \pm \sigma \alpha_{\Gamma}$. It means that with increasing temperature, particles can be emitted further and further away from the time at which the level crosses the Fermi energy of the reservoirs. Note, however, that a small symmetric contribution always persists, guaranteeing thatalso at high temperatures-the average energy carried per pulse is positive and given by $D \mathcal{E}$.

\section{SPECTRAL CURRENTS}

The spectral current allows to access the energetic distribution of emitted particles. It can be measured by inserting scatterers with a specifically designed energy-dependent transmission into a setup $[8,62,63]$ or from measurements of the charge current noise in the presence of an appropriately tuned bias voltage $[17,31]$. The spectral current is given by

$$
i(E)=D \sum_{n=-\infty}^{\infty}\left|S\left(E, E_{n}\right)\right|^{2}\left[f_{\mathrm{L}}\left(E_{n}\right)-f_{\mathrm{R}}(E)\right] .
$$

Here, for the bias-voltage driven system and the locally modulated edge state (setups B and C) the absolue value squared of the scattering matrix has to be replaced by the energyindependent probabilities $\left|c_{\mathrm{L}, n}\right|^{2}$ from Eq. (18) and $\left|c_{\mathrm{g}, n}\right|^{2}$ from Eq. (23), respectively. However, also for the mesoscopic capacitor, we get from Eq. (4) for $n \neq 0$

$$
\left|S_{\text {cap }}\left(E, E_{n}\right)\right|^{2}=(2 \Omega \sigma)^{2} e^{-2|n| \Omega \sigma}=:\left|S_{n}\right|^{2},
$$

which is energy independent and equal to $\left|c_{\mathrm{g}, n}\right|^{2}$ and to $\left|c_{\mathrm{L}, n}\right|^{2}$ for $n \geqslant 0$. It means that (in the regime considered here) the spectral currents of the three sources are almost identical at all temperatures, except for the fact that in the leviton case, only electrons are emitted. Technically, in Eq. (39), this difference

\footnotetext{
${ }^{4}$ For the injection of a hole (absorption of an electron) in the second half of the cycle, an equivalent feature is found, see Fig. 3(c): particles absorbed at positive/negative energies lead to negative/positive energy currents.
}

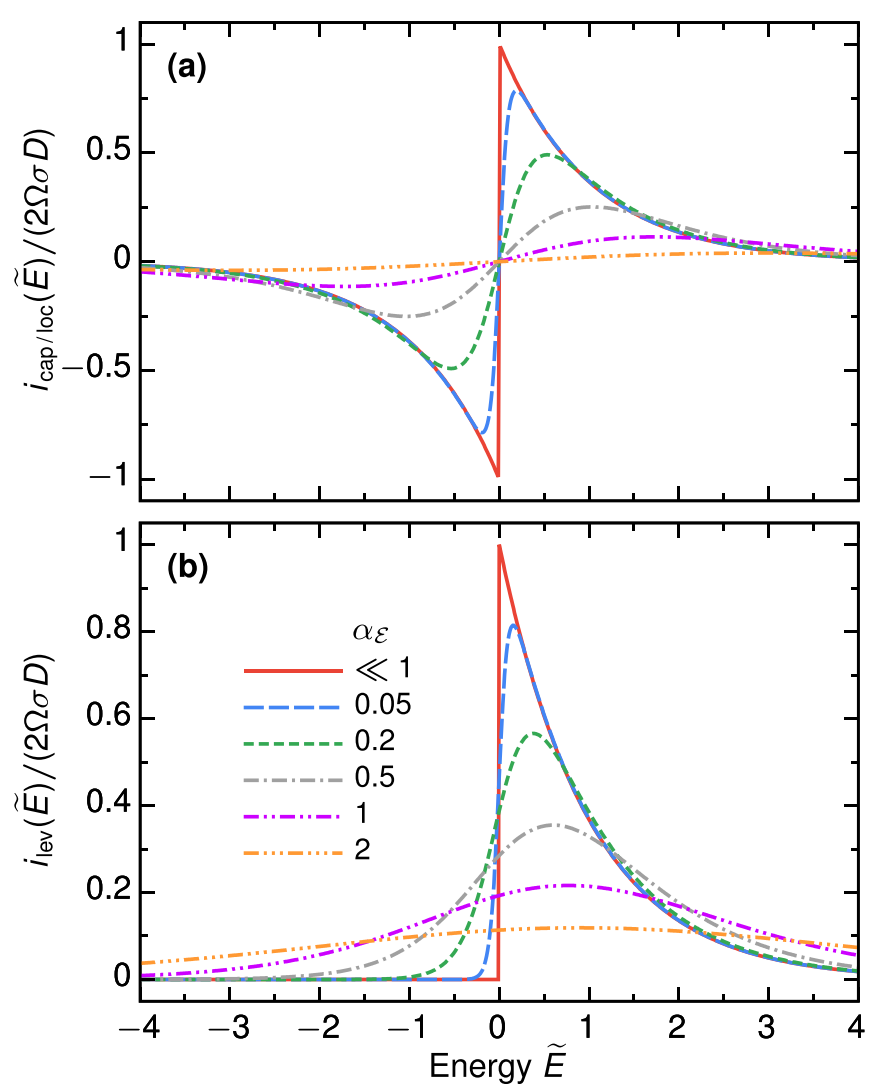

FIG. 4. Spectral current emitted (a) from a time-dependently driven capacitor (setup A) or a locally modulated edge state (setup C), and (b) from a Lorentzian-shaped bias voltage (setup B) as a function of the dimensionless energy $\widetilde{E}=E / \mathcal{E}$ for different values of $\alpha_{\mathcal{E}}=k_{\mathrm{B}} T / \mathcal{E}$.

arises from the vanishing contributions to $\left|c_{\mathrm{L}, n}\right|^{2}$ for negative $n$ and the constant part of the driving $\bar{V}_{\mathrm{b}}=\hbar \Omega /(-e)$ entering the Fermi function $f_{\mathrm{L}}\left(E_{n}\right)$, where it leads to an effective shift of the energy index $f_{\mathrm{L}}\left(E_{n}\right) \rightarrow f\left(E_{n-1}\right)$. We find that this behavior is in strong contrast to the time-resolved currents, which, as discussed in the previous section, were shown to have very different temperature-dependent characteristics.

A general evaluation of the sum in Eq. (39), see Appendix E, shows that the energy scale of the temperature only enters the spectral current through the factor $\alpha_{\mathcal{E}}=k_{\mathrm{B}} T / \mathcal{E}$. More generally, the energy $\mathcal{E}$ set by the driving, see Eq. (13), turns out to be the relevant energy scale of the energy-resolved current, which we therefore always express in terms of the dimensionless energy $\widetilde{E}=E / \mathcal{E}$ in this section. Figure 4 illustrates the evolution of the spectral current with changing $\alpha_{\mathcal{E}}$. The analytical results for the limiting cases for small and large temperatures elucidate the shape of the shown curves. In the limit of vanishingly small temperatures, $\alpha_{\mathcal{E}} \ll 1$, the spectral current as a function of the dimensionless energy $\widetilde{E}=E / \mathcal{E}$ is given by

$$
\begin{aligned}
i_{\text {lev }}(\widetilde{E}) & =2 \Omega \sigma D e^{-|\widetilde{E}|}[\theta(\widetilde{E})], \\
i_{\text {cap } / \mathrm{loc}}(\widetilde{E}) & =2 \Omega \sigma D e^{-|\widetilde{E}|}[\theta(\widetilde{E})-\theta(-\widetilde{E})] .
\end{aligned}
$$

At low temperatures, the shape of the spectral current is, thus, an exponential, see, e.g., Ref. [55], with a width in 
energy given by $\mathcal{E}$. In the opposite limit of high temperatures, $\alpha_{\mathcal{E}} \gg 1$, we find

$$
\begin{aligned}
i_{\mathrm{lev}}(\widetilde{E}) & =2 \Omega \sigma D\left[-\frac{\partial}{\partial \widetilde{E}}+2 \frac{\partial^{2}}{\partial \widetilde{E}^{2}}\right] \frac{1}{1+e^{\widetilde{E} / \alpha_{\mathcal{E}}}}, \\
i_{\text {cap } / \mathrm{loc}}(\widetilde{E}) & =2 \Omega \sigma D\left[2 \frac{\partial^{2}}{\partial \widetilde{E}^{2}}\right] \frac{1}{1+e^{\widetilde{E} / \alpha_{\mathcal{E}}}},
\end{aligned}
$$

namely, a rounding of the signal with the shape dictated by derivatives of the Fermi function.

The conclusion from these results is that while the timeresolved current emitted from the bias driving and the local modulation of an edge state (setups B and C) are not affected by temperature, the time-integrated, energy-resolved currents (i.e., the spectral currents) are actually strongly affected. Nevertheless, the average energy transported by a single-particle pulse emitted from any of the three sources is not altered, $\int d t I^{\mathcal{E}}(t)=\mathcal{E}^{2} \mathcal{T} \int d \widetilde{E} E i(\widetilde{E})=D \mathcal{E}$, independent of temperature.

\section{ZERO-FREQUENCY NOISE}

In this section, we study zero-frequency noise of charge and energy currents, as well as the mixed charge-energy current noise. In general, the study of correlation functions of this type yields additional information about a setup. This important observable has indeed been heavily exploited in the field of quantum optics with electrons based on singleelectron sources. One reason is that the charge-current noise is a measure of the precision of the source [53,64-72]. But the charge-current noise has also been used to identify correlations in Hanbury Brown-Twiss and Hong-Ou-Mandel setups or interferometer setups. With the help of the latter setups also the energy distributions of injected signals could be determined. Recently, measurements of the noise have even been performed for tomography with the aim to reconstruct the full single-particle coherence $[16,19,27]$. Also the energy(or heat-) current noise [40,41], or even the mixed noise between charge and energy currents [42], have recently been suggested as a tool to analyze setups with single-electron sources. Here, we investigate how these different types of noise are influenced by temperature effects and demonstrate that-except for the mixed noise- the results do not depend on the type of a source from which particles are injected.

\section{A. Charge-current noise}

The charge-current noise of the mesoscopic capacitor is found to be

$$
\begin{aligned}
\mathcal{P}_{\text {cap }}^{I I}= & \frac{2 e^{2}}{h} D^{2} k_{\mathrm{B}} T \\
& +\frac{e^{2}}{h} D(1-D) \sum_{n=-\infty}^{\infty}\left|S_{n}\right|^{2} n \hbar \Omega \operatorname{coth}\left(\frac{n \hbar \Omega}{2 k_{\mathrm{B}} T}\right) .
\end{aligned}
$$

Note that only the energy-independent absolute value of the scattering matrix enters here. The same equation holds for the locally modulated edge state, where the absolute value squared of the scattering matrix is identical to the one of the driven mesoscopic capacitor. Employing an equivalent argument as in Sec. IV, we find the same result for the chargecurrent noise of the leviton emission with the only difference that only positive $n$ contribute to the sum in Eq. (43).

The similarity of the results for the three different sources stems from the fact that the zero-frequency noise averages out the effect of timescales of the order of the wave-packet size. An influence of the level broadening of the mesoscopic capacitor, entering time-resolved currents through the parameter $\alpha_{\Gamma}=k_{\mathrm{B}} T / \Gamma$, on the zero-frequency noise of these sources emitting well-separated particle pulses, is therefore not expected. The following calculation will confirm this, not only for the charge current noise but also for the energy-current noise and mixed noise. In what follows, we will carefully analyze how temperature affects the noise of all three studied single-particle sources and point out crucial differences due to differences in the injected number and polarity of particles.

In the zero-temperature limit, Eq. (43) yields the wellknown result for the shot noise [21,64],

$$
\mathcal{P}^{I I}\left(k_{\mathrm{B}} T \rightarrow 0\right) \equiv \mathcal{P}_{0 \text {-sh }}^{I I}=\frac{e^{2}}{\mathcal{T}} D(1-D) N,
$$

with $N$ denoting the number of injected particles. This number equals $N=1$ for the leviton-injection, whereas $N=2$ for the driven mesoscopic capacitor and the locally modulated edge state. For temperatures different from zero (namely, larger than the smallest energy scale of the problem, $k_{\mathrm{B}} T>\hbar \Omega$ ), we find

$$
\mathcal{P}^{I I}=\mathcal{P}_{\text {th }}^{I I}+\mathcal{P}_{0 \text {-sh }}^{I I}+\mathcal{P}_{T \text {-sh }}^{I I} .
$$

The three contributions to Eq. (44b) are the well-known thermal noise

$$
\mathcal{P}_{\text {th }}^{I I}=\frac{2 e^{2}}{h} D k_{\mathrm{B}} T,
$$

which is independent of the driving, the zero-temperature shot noise, $\mathcal{P}_{0 \text {-sh }}^{I I}$ as given in Eq. (44a), and a third contribution arising from shot noise at finite temperatures

$$
\mathcal{P}_{T \text {-sh }}^{I I}=\frac{2 e^{2}}{\mathcal{T}} D(1-D) N\left[\alpha_{\mathcal{E}}^{2} \psi^{(1)}\left(\alpha_{\mathcal{E}}\right)-1\right],
$$

with $\psi^{(n)}(z)$ being the polygamma function of order $n$.

In Fig. 5, we show the full charge-current noise $\mathcal{P}_{\text {cap }}^{I I}$ for the mesoscopic capacitor (i.e., with $N=2$, solid gray line), as well as its separate contributions as decomposed in Eqs. (44). Except for the thermal noise, which is driving independent, $\mathcal{P}_{\text {cap }}^{I I}$ depends on temperature uniquely through the parameter $\alpha_{\mathcal{E}}=k_{\mathrm{B}} T / \mathcal{E}$, ${ }^{5}$ which compares the energy scale set by temperature, $k_{\mathrm{B}} T$, to the energy scale set by the driving, $\mathcal{E}$. We choose this parameter $\alpha_{\mathcal{E}}$ as the plotting parameter for all plots of zero-frequency noise presented in this section. As long as $2 k_{\mathrm{B}} T<\hbar \Omega$ (or equivalently $\alpha_{\mathcal{E}}<\Omega \sigma$ ), the thermal noise plays no important role with respect to the zero-temperature shot noise, which is fully induced by the driving. The shot

\footnotetext{
${ }^{5}$ As expected, the broadening of the capacitor level is not a relevant energy scale for the shot noise in the single-particle emission regime considered in this paper, since the (energy-dependent) emission times do not enter the noise expression.
} 


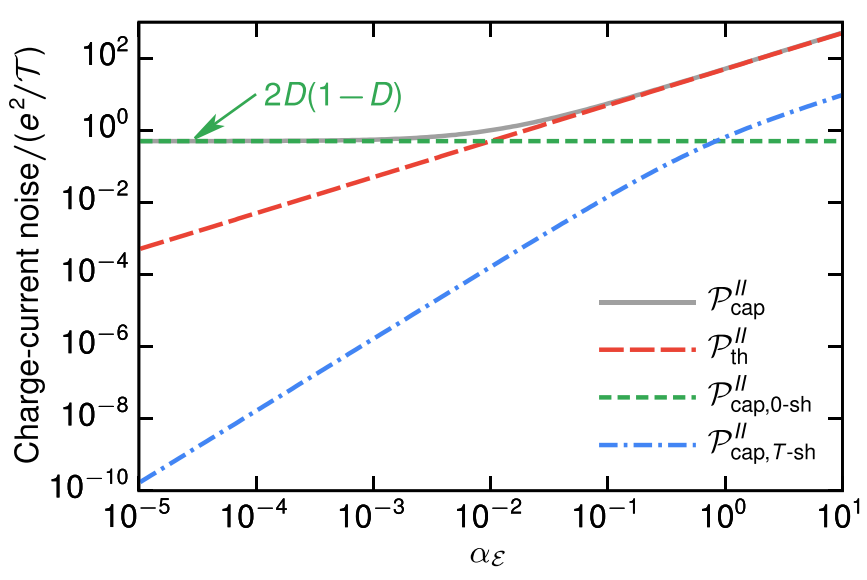

FIG. 5. Charge-current noise of the driven mesoscopic capacitor (setup A), which is identical to the one of setup $\mathrm{C}$, shown as a function of $\alpha_{\mathcal{E}}=k_{\mathrm{B}} T / \mathcal{E}$. Importantly, for setup B only the thermal contribution $\mathcal{P}_{\text {th }}^{I I}$ is the same, whereas the driving-dependent contributions, $\mathcal{P}_{0 \text {-sh }}^{I I}$ and $\mathcal{P}_{T \text {-sh }}^{I I}$, differ by an overall factor 2 . Three separate contributions, following Eq. (44b), are shown additionally. We choose $D=0.5$ and $\Omega \sigma=0.01$.

noise at finite temperatures exceeds the zero-temperature contribution only for $\alpha_{\mathcal{E}}>1$. However, in the resonant limit, $\Omega \sigma \ll 1$, this crossover takes place solely in a regime, where the thermal noise is already the dominant noise contribution.

\section{B. Energy-current noise}

The energy-current noise of a time-dependently driven system, in general, consists of a transport contribution, which in the zero-temperature limit yields the shot noise, and an interference contribution [40,53]. The latter-in contrast to the interference part of the charge-current noise-depends on the driving and is nonzero even at zero temperatures. The general expression for the energy-current noise of the capacitor written in terms of scattering matrices, equivalent to Eq. (43), is lengthy, and hence, included only in Appendix F. However, there, we also show that, in the single-particle emission regime considered here, both contributions are independent of the (energy-dependent) emission times of the scattering matrix. This confirms the statement made in Sec. V A, namely, that the (energy- and charge-current) noise do not depend on the specific time-resolved shape of the emitted current pulses. As a result, not only the charge-current noise, but also the energy-current noise of the three sources discussed in this paper differ from each other only due to the number of injected particles.

We now analyze the temperature-dependence of the energy-current noise. In the zero-temperature limit, one obtains

$$
\begin{aligned}
\mathcal{P}^{\mathcal{E} \mathcal{E}}\left(k_{\mathrm{B}} T \rightarrow 0\right) & \equiv \mathcal{P}_{0 \text {-int }}^{\mathcal{E} \mathcal{E}}+\mathcal{P}_{0 \text {-sh }}^{\mathcal{E} \mathcal{E}} \\
& =\frac{\mathcal{E}^{2}}{\mathcal{T}} D^{2} N+\frac{\mathcal{E}^{2}}{\mathcal{T}} 2 D(1-D) N,
\end{aligned}
$$

where the first term is the zero-temperature interference contribution and the second one corresponds to the zerotemperature shot noise stemming from the transport part.

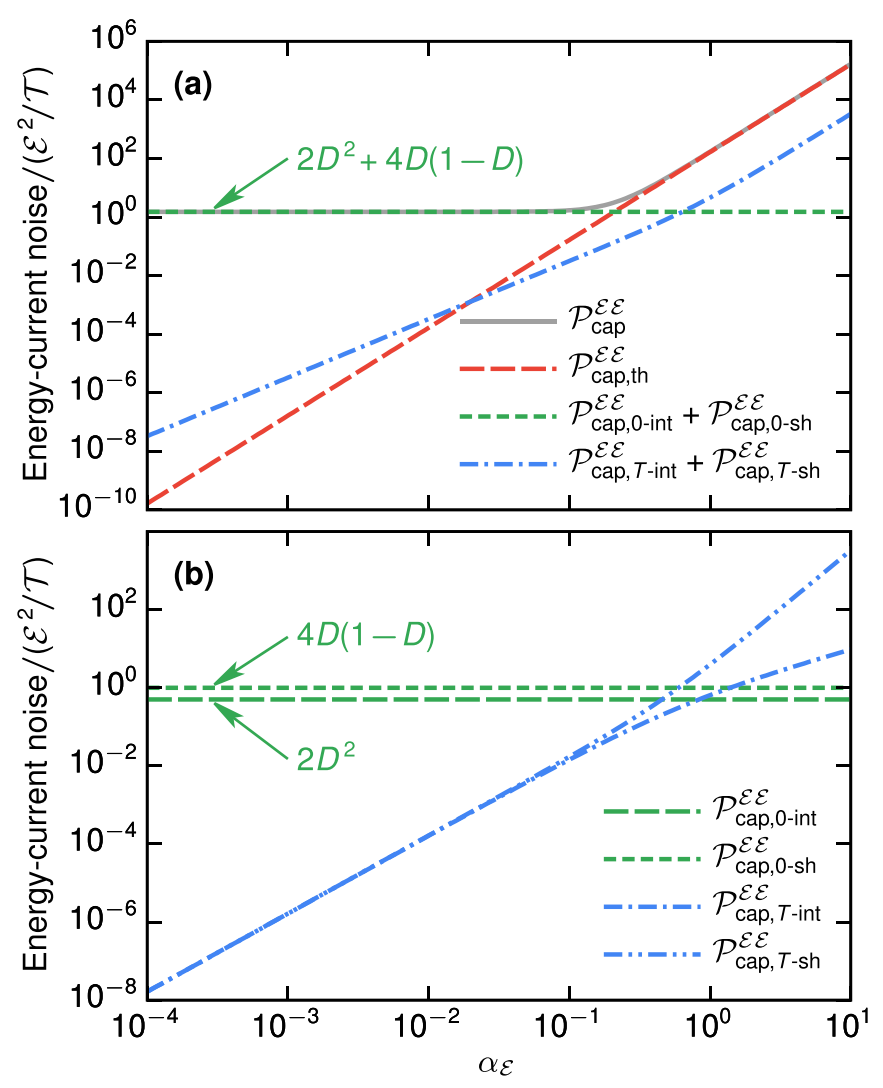

FIG. 6. Energy-current noise of the driven mesoscopic capacitor (setup A), which is identical to the one of setup C, plotted as a function of $\alpha_{\mathcal{E}}=k_{\mathrm{B}} T / \mathcal{E}$. Importantly, for setup B, only the thermal contribution $\mathcal{P}_{\text {th }}^{\mathcal{E}}$ is the same, whereas all remaining, drivingdependent contributions differ by an overall factor 2 . Separate contributions, following Eqs. (45), are shown additionally. (a) presents the pure thermal noise and the zero-temperature as well as the finitetemperature contributions of the combined transport and interference part. (b) illustrates the decomposition of the transport (shot) and interference part separately. We choose $D=0.5$ and $\Omega \sigma=0.01$.

When temperature gets larger $\left(k_{\mathrm{B}} T>\hbar \Omega\right)$, we find

$$
\mathcal{P}^{\mathcal{E} E}=\mathcal{P}_{\text {th }}^{\mathcal{E E}}+\mathcal{P}_{0 \text {-int }}^{\mathcal{E E}}+\mathcal{P}_{0 \text {-sh }}^{\mathcal{E}}+\mathcal{P}_{T \text {-int }}^{\mathcal{E E}}+\mathcal{P}_{T \text {-sh }}^{\mathcal{E} \mathcal{E}} .
$$

Thermal noise (namely, the driving-independent part of the interference contribution) is given by

$$
\mathcal{P}_{\text {th }}^{\mathcal{E E}}=\frac{2 \pi^{2}}{3 h} D\left(k_{\mathrm{B}} T\right)^{3} .
$$

In addition, both the interference and the transport part get driving-dependent thermal contributions

$$
\begin{aligned}
\mathcal{P}_{T \text {-int }}^{\mathcal{E} \mathcal{E}}= & \frac{2 \mathcal{E}^{2}}{\mathcal{T}} D^{2} N\left[\alpha_{\mathcal{E}}^{2} \psi^{(1)}\left(\alpha_{\mathcal{E}}\right)-1\right], \\
\mathcal{P}_{T \text {-sh }}^{\mathcal{E} \mathcal{E}}= & \frac{2 \mathcal{E}^{2}}{3 \mathcal{T}} D(1-D) N\left\{\alpha_{\mathcal{E}}^{4} \psi^{(3)}\left(\alpha_{\mathcal{E}}\right)-6\right. \\
& \left.+\pi^{2} \alpha_{\mathcal{E}}^{2}\left[\alpha_{\mathcal{E}}^{2} \psi^{(1)}\left(\alpha_{\mathcal{E}}\right)-1 / 2\right]\right\} .
\end{aligned}
$$

In Fig. 6, we show the full energy-current noise, as well as its separate contributions. The main contribution to the total energy-current noise [plotted in panel (a) as the solid gray line] is given by the sum of zero-temperature transport 
and interference parts [plotted in panel (a) as the finely dashed green line] up to temperatures fulfilling the condition $2\left(k_{\mathrm{B}} T\right)^{3} \approx \mathcal{E}^{2} / \mathcal{T}$, or equivalently $\alpha_{\mathcal{E}} \approx \sqrt[3]{\Omega \sigma}$. It means that the parameter $\alpha_{\mathcal{E}}$, at which thermal noise starts to play a role is much larger than for the charge-current noise. The driving-dependent thermal contributions for the energy current become relevant at $\alpha_{\mathcal{E}} \approx 1$, but stay well below the pure thermal noise. This behavior is analogous to that found for the charge-current noise. Finally, all the transport and interference contributions are plotted separately in Fig. 6(b). One can observe there, that the transport part is much more affected by temperature than the interference part. We note that a separate readout of transport and interference contributions is enabled by their different $D$ dependence.

\section{Mixed noise}

Last but not least, we present results for the mixed zerofrequency correlator of charge and energy currents. The mixed noise between charge and energy has been addressed little so far [73-75], in particular, in time-dependently driven systems as the ones considered here $[39,42,44,45]$. It yields a measure for the amount of correlations between charge and energy flow; here we show that it constitutes a further observable for spectroscopy for only one of the studied single-particle source implementations. The general expression for the mixed noise from the driven capacitor and the locally modulated edge state is given by

$$
\mathcal{P}_{\text {cap }}^{I \mathcal{E}}=\frac{-e}{2 h} D(1-D) \sum_{n=-\infty}^{\infty}\left|S_{n}\right|^{2}(n \hbar \Omega)^{2} \operatorname{coth}\left(\frac{n \hbar \Omega}{2 k_{\mathrm{B}} T}\right) .
$$

Due to the pure ac driving of setups A and C, going along with the emission of an equal number of electrons and holes, the mixed noise vanishes identically, $\mathcal{P}_{\text {cap } / \text { loc }}^{I \mathcal{E}} \equiv 0$. Technically, this arises from the fact that the first two factors in the sum in Eq. (46) are even, see also Eq. (40), while the third one is odd. Instead, for the leviton emission [42], as realized in setup B, one finds a finite contribution due to the asymmetry induced by the pure electron emission (or equivalently, by the nonvanishing zero-component of the bias). We can hence conclude that zero-frequency mixed noise only arises in the presence of a dc bias voltage, namely, when the number of emitted electrons and holes differ from each other. In the zerotemperature limit, the shot noise contribution to the mixed noise reads

$$
\mathcal{P}^{I \mathcal{E}}\left(k_{\mathrm{B}} T \rightarrow 0\right) \equiv \mathcal{P}_{0 \text {-sh }}^{I \mathcal{E}}=\frac{-e \mathcal{E}}{\mathcal{T}} D(1-D) \delta N,
$$

with the difference between injected electrons and holes: $\delta N=1$ (setup B) and $\delta N=0$ (setup A and C). For finite temperatures $\left(k_{\mathrm{B}} T>\hbar \Omega\right)$, there are only two additional contributions to the mixed noise,

$$
\mathcal{P}^{I \mathcal{E}}=\mathcal{P}_{0 \text {-sh }}^{I \mathcal{E}}+\mathcal{P}_{T \text {-sh }}^{I \mathcal{E}}+\mathcal{P}_{T \text {-int }}^{I \mathcal{E}} .
$$

We see that there is no pure thermal contribution. The full interference contribution is both temperature- and drivingdependent,

$$
\mathcal{P}_{T \text {-int }}^{I \mathcal{E}}=\frac{e^{2}}{h} D^{2} \delta N \bar{V}_{\mathrm{b}} k_{\mathrm{B}} T=\frac{-e \mathcal{E}}{\mathcal{T}} D^{2} \delta N \alpha_{\mathcal{E}}
$$

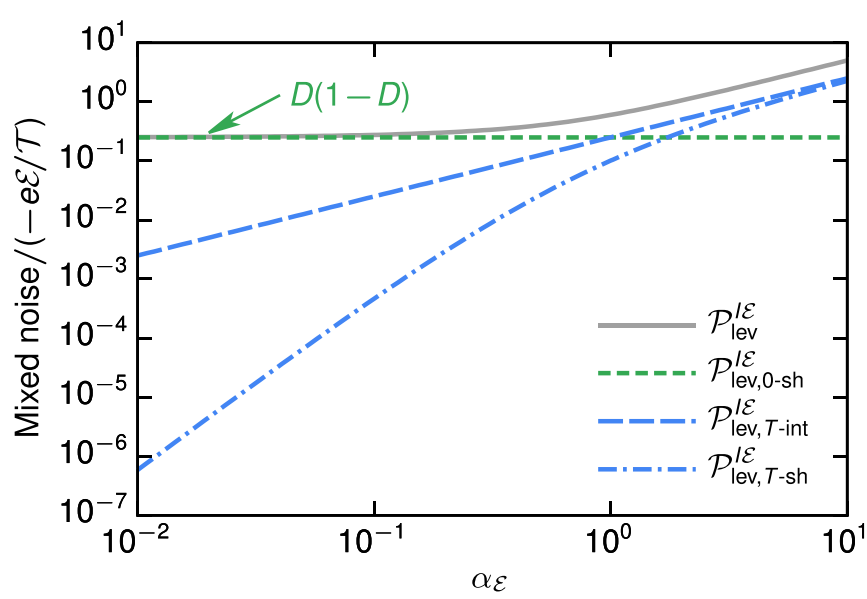

FIG. 7. Mixed charge-energy current noise, plotted as a function of $\alpha_{\mathcal{E}}=k_{\mathrm{B}} T / \mathcal{E}$, for the Lorentzian-shaped bias voltage driving (setup B) leading to emission of levitons. We choose $D=0.5$ and $\Omega \sigma=0.01$.

with $\bar{V}_{\mathrm{b}}=\hbar \Omega /(-e)$. The thermal contribution to the shot noise (transport part) is given by

$$
\mathcal{P}_{T \text {-sh }}^{I \mathcal{E}}=\frac{-e \mathcal{E}}{\mathcal{T}} D(1-D) \delta N\left[-\alpha_{\mathcal{E}}^{3} \psi^{(2)}\left(\alpha_{\mathcal{E}}\right)-1\right] .
$$

Figure 7 presents the full mixed noise and its separate contributions. As for the other noise expressions, the drivingdependent thermal transport and interference parts of $\mathcal{P}_{\mathrm{lev}}^{I \mathcal{E}}$ contribute with equal weight starting from $\alpha_{\mathcal{E}} \approx 1$.

\section{CONCLUSIONS AND OUTLOOK}

We have analyzed the characteristics of time-resolved charge and energy, as well as spectral currents, together with the zero-frequency correlators of charge and energy currents of single-electron sources, which emit noiseless singleparticle pulses as minimal excitations of the Fermi sea. The main focus is on how these observables vary for different types of single-electron source and on the analysis of the temperature dependence of the observables. The relevant types of single-electron sources, which we consider in this paper are as follows: (setup A) a driven mesoscopic capacitor emitting single particles due to a pure ac gate driving of a confined region with a discrete energy spectrum; (setup B) leviton emission taking place due to a time-dependent bias voltage (with a nonvanishing dc component $\bar{V}_{\mathrm{b}}$ ) in the absence of any confinement in the conductor; (setup C) an emission scheme not relying on the confinement but allowing for electron and hole emission due to a local modulation of an edge state.

The inherent properties of these sources lead to the following observable effects in the analyzed quantities. First of all, due to the discrete spectrum of the driven capacitor, leading to an energy dependence of its scattering matrix, the time-resolved charge and energy currents for such a source are strongly temperature-dependent. In particular, the ratio between the driving-independent level broadening $\Gamma$, Eq. (11), and temperature plays an important role here. This behavior is in contrast to that for the leviton emission and the locally 
modulated edge state, where temperature has no impact on time-resolved currents.

Furthermore, when looking at spectral currents and zerofrequency noises, the discrete spectrum of the mesoscopic capacitor leading to an energy dependence of the scattering matrix plays no role. Instead, it is the energy dependence of the Fermi functions, which entirely determines temperaturedependent effects. Indeed, the temperature dependence of the spectral current and noises is fully governed by the ratio between driving dependent quantities, namely, the frequency $\Omega$ or the energy emitted per pulse $\mathcal{E}$, as compared to temperature. This strong temperature-dependence is carefully analyzed in this manuscript. Major differences in the observed quantities for different sources then result from the number of emitted particles and from the spectral weight of particles at positive and negative energies (differing for electrons and holes). Interestingly, the mixed charge-energy current noise has a special role: it requires a nonvanishing dc component of the bias $\bar{V}_{\mathrm{b}}$, and thus, it vanishes identically in setups $\mathrm{A}$ and $\mathrm{C}$, where electrons are emitted from a pure ac gate-driving.

These fundamental properties of the major observables used for analysis in quantum optics with electrons, which can be ascribed to variations of a small set of relevant parameters $\left(\Omega \sigma, \alpha_{\Gamma}\right.$, and $\left.\alpha_{\mathcal{E}}\right)$, are expected to be important for future studies of setups containing any of the three single-particle sources analyzed in this paper. Specifically, it will help to distinguish the fundamental source properties, analyzed in this paper, from other features arising in complex setups, fed by these single-particle sources. These latter features could result from interaction effects leading to decoherence during propagation along edge states, from controlled two-particle effects in interferometers with several synchronized sources, or from scattering at impurities, to give some examples.

\section{ACKNOWLEDGMENTS}

Funding from the Knut and Alice Wallenberg Foundation through the Academy Fellow program (J.S., N.D. and M.M.), from the EU ITN PhD4Energy, Grant No. 608153 (S.K.), and from the Swedish VR is gratefully acknowledged.

\section{APPENDIX A: CURRENTS AND NOISE-DEFINITIONS}

In this Appendix, we define the charge and energy current operators, their expectation values, and their correlations. These expressions can also be found in textbooks and reviews, such as, Refs. [55,76]. For the charge and energy current operators in contact $\alpha$, we have

$$
\hat{I}_{\alpha}(t)=-\frac{e}{h} \int_{-\infty}^{\infty} d E d E^{\prime} \hat{i}_{\alpha}\left(E, E^{\prime}\right) e^{i\left(E-E^{\prime}\right) t / \hbar},
$$

with $e>0$, as well as

$$
\hat{I}_{\alpha}^{\mathcal{E}}(t)=\frac{1}{h} \int_{-\infty}^{\infty} d E d E^{\prime} \frac{E+E^{\prime}}{2} \hat{i}_{\alpha}\left(E, E^{\prime}\right) e^{i\left(E-E^{\prime}\right) t / \hbar} .
$$

The operator $\hat{i}_{\alpha}\left(E, E^{\prime}\right)=\hat{b}_{\alpha}^{\dagger}(E) \hat{b}_{\alpha}\left(E^{\prime}\right)-\hat{a}_{\alpha}^{\dagger}(E) \hat{a}_{\alpha}\left(E^{\prime}\right)$ contains creation and annihilation operators for incoming $\left[\hat{b}_{\alpha}^{\dagger}(E) \hat{b}_{\alpha}\left(E^{\prime}\right)\right]$ and outgoing $\left[\hat{a}_{\alpha}^{\dagger}(E) \hat{a}_{\alpha}\left(E^{\prime}\right)\right]$ fluxes in contact $\alpha$. For simplicity, we restrict the present analysis to the case of a single transport channel, which can be extended to the general multi-channel case, e.g., as done in Ref. [40]. The incoming and outgoing scattering states are related to each other through a scattering matrix

$$
\hat{b}_{\alpha}\left(E_{n}\right)=\sum_{\beta=\mathrm{L}, \mathrm{R}} \sum_{m=-\infty}^{\infty} S_{\alpha \beta}\left(E_{n}, E_{m}\right) \hat{a}_{\beta}\left(E_{m}\right),
$$

with $E_{m} \equiv E+m \hbar \Omega$, and $m \hbar \Omega$ (for $m \in \mathbb{Z}$ ) standing for the amount of absorbed or emitted energy quanta in a scattering process. See the main text for specific expressions.

In order to obtain the time-resolved charge and energy currents, $I_{\alpha}(t)$ and $I_{\alpha}^{\mathcal{E}}(t)$, the expectation values of Eqs. (A1) and (A2), respectively, have to be calculated

$$
I_{\alpha}(t)=\left\langle\hat{I}_{\alpha}(t)\right\rangle \quad \text { and } \quad I_{\alpha}^{\mathcal{E}}(t)=\left\langle\hat{I}_{\alpha}^{\mathcal{E}}(t)\right\rangle,
$$

where $\langle\ldots\rangle$ denotes the quantum statistical average. Moreover, the time-averaged charge and energy currents, $\bar{I}_{\alpha}$ and $\bar{I}_{\alpha}^{\mathcal{E}}$, can be derived by integrating Eq. (A4) over one period $\mathcal{T}$, that is, $\bar{I}_{\alpha}=\int_{0}^{\mathcal{T}} d t\left\langle\hat{I}_{\alpha}(t)\right\rangle / \mathcal{T}$ and $\bar{I}_{\alpha}^{\mathcal{E}}=\int_{0}^{\mathcal{T}} d t\left\langle\hat{I}_{\alpha}^{\mathcal{E}}(t)\right\rangle / \mathcal{T}$.

The fluctuations of charge and energy currents are accessible through the (generalized) zero-frequency noise,

$$
\mathcal{P}_{\alpha \beta}^{X Y}=\int_{0}^{\mathcal{T}} \frac{d t}{\mathcal{T}} \int_{-\infty}^{\infty} d t^{\prime}\left\langle\Delta X_{\alpha}(t) \Delta Y_{\beta}\left(t+t^{\prime}\right)\right\rangle
$$

The current fluctuation, $\Delta \hat{X}(t)=\hat{X}(t)-\langle\hat{X}(t)\rangle$, is kept general here, and will in the following be replaced by the fluctuations of the relevant current operators, $\hat{I}$ or $\hat{I}^{\mathcal{E}}$.

\section{APPENDIX B: GENERAL EXPRESSIONS FOR CURRENTS AND NOISES}

Here, we present general expressions for the currents and their correlators describing a situation where both timedependent driving of the contacts, as well as a time-dependent scatterer, such as the driven mesoscopic capacitor or the locally modulated edge state, can be present in the same setup. For this reason, we incorporate both the Floquet scattering matrix of the central region, $S_{\alpha \beta}\left(E_{n}, E_{m}\right)$, as well as coefficients accounting for the driving of the bias voltage, $c_{\alpha, m}$ with $\alpha=$ L or R. Employing Eqs. (A1) and (A2), we find the general expression for the time-resolved charge and energy currents to be

$$
I_{\alpha}^{(\mathcal{E})}(t)=\sum_{n=-\infty}^{\infty} e^{-i n \Omega t} I_{\alpha, n}^{(\mathcal{E})}
$$

with $^{6}$

$$
\begin{aligned}
\left(\begin{array}{c}
I_{\alpha, n} \\
I_{\alpha, n}^{\mathcal{E}}
\end{array}\right)= & \frac{1}{h} \sum_{\beta} \sum_{m, q=-\infty}^{\infty} \int d E\left(\begin{array}{c}
-e \\
E+\frac{n \hbar \Omega}{2}
\end{array}\right) \\
& \times\left\{S_{\alpha \beta}^{*}\left(E, E_{m}\right) S_{\alpha \beta}\left(E_{n}, E_{m+q}\right) \mathcal{F}_{\beta, q}\left(E_{m}\right)\right. \\
& \left.-S_{\alpha \beta}^{*}\left(E, E_{m}\right) S_{\alpha \beta}\left(E_{n-q}, E_{m}\right) \mathcal{F}_{\alpha, q}(E)\right\},
\end{aligned}
$$

\footnotetext{
${ }^{6}$ Note that we make the following assumption for current (and noise) measurements in setups with time-dependent bias driving: the detection of the signal is always assumed to take place before particles scattered at the central scattering region enter again the region of time-dependent bias driving.
} 
with the auxiliary function $\mathcal{F}_{\alpha, q}(E)$ defined as

$$
\mathcal{F}_{\alpha, q}(E)=\sum_{l=-\infty}^{\infty} c_{\alpha, l}^{*} c_{\alpha, l+q} f_{\alpha}\left(E_{-l}\right) .
$$

Note that $\mathcal{F}_{\alpha, q}(E)$ has a clear physical interpretation only for $q=0$, representing then an effective nonequilibrium distribution function induced by the ac driving in contact $\alpha$. This function,

$$
\tilde{f}_{\alpha}(E) \equiv \mathcal{F}_{\alpha, 0}(E)=\sum_{l=-\infty}^{\infty}\left|c_{\alpha, l}\right|^{2} f_{\alpha}\left(E_{-l}\right),
$$

takes into account the fact that in the presence of the ac driving also states originating from energies, which differ from $E$ by an integer multiple of $\hbar \Omega$, contribute to transport at energy $E$ [40].

Furthermore, from Eqs. (B1) and (B2), one can obtain the time-averaged currents, corresponding to the term for $n=0$ in the series (B1),

$$
\bar{I}_{\alpha}^{(\mathcal{E})}=\int_{0}^{\mathcal{T}} \frac{d t}{\mathcal{T}} I_{\alpha}^{(\mathcal{E})}(t)=I_{\alpha, 0}^{(\mathcal{E})} .
$$

An equivalent procedure for deriving the time-averaged currents involves calculation of energy integrals over a spectral current $i_{\alpha}(E)$, that is, $\bar{I}_{\alpha}=-e \int d E i_{\alpha}(E) / h$, and $\bar{I}_{\alpha}^{\mathcal{E}}=$ $\int d E E i_{\alpha}(E) / h$, with

$$
\begin{aligned}
i_{\alpha}(E)= & \sum_{\beta} \sum_{m, q=-\infty}^{\infty} S_{\alpha \beta}^{*}\left(E, E_{m}\right) S_{\alpha \beta}\left(E, E_{m+q}\right) \\
& \times\left[\mathcal{F}_{\beta, q}\left(E_{m}\right)-\delta_{q 0} \mathcal{F}_{\alpha, q}(E)\right] .
\end{aligned}
$$

While the previous equations for the current operators, Eqs. (A1) and (A2), were kept on a very general level, here we have explicitly evaluated expectation values of the occupations of incoming current states, leading to the occurrence of the Fermi function $f_{\alpha}(E)=\left\{1+\exp \left[\left(E-\mu_{\alpha}\right) /\left(k_{\mathrm{B}} T\right)\right]\right\}^{-1}$.

Note that the time-resolved charge current and the spectral current are two different marginals of the so-called Wigner function $[59,77] W_{\alpha}(\bar{t}, E)$ :

$$
\begin{aligned}
& I_{\alpha}(\bar{t})=e \int d E W_{\alpha}(\bar{t}, E), \\
& i_{\alpha}(E)=h \int d \bar{t} W_{\alpha}(\bar{t}, E) .
\end{aligned}
$$

The Wigner function is given by

$$
W_{\alpha}(\bar{t}, E)=\int d \tau e^{i E \tau / \hbar} \mathcal{G}_{\alpha}(\bar{t}+\tau / 2 ; \bar{t}-\tau / 2),
$$

which is the partial Fourier transform of the first order electronic correlation function (Glauber function), $\mathcal{G}_{\alpha}\left(t^{\prime} ; t\right)$ [16] with respect to the time difference $\tau=t-t^{\prime}$ at the average time $\bar{t}=\left(t+t^{\prime}\right) / 2$. This correlation function for emitted particles from any of the three single-particle sources discussed here can be written as

$$
\begin{aligned}
\mathcal{G}_{\alpha}\left(t ; t^{\prime}\right)= & \sum_{\beta} \sum_{m, n, q} \frac{1}{h} \int d E e^{i E t / \hbar} e^{-i E_{n} t^{\prime} / \hbar} \\
& \times\left\{S_{\alpha \beta}^{*}\left(E, E_{m}\right) S_{\alpha \beta}\left(E_{n}, E_{m+q}\right) \mathcal{F}_{\beta, q}\left(E_{m}\right)\right. \\
& \left.-S_{\alpha \beta}^{*}\left(E, E_{m}\right) S_{\alpha \beta}\left(E_{n-q}, E_{m}\right) \mathcal{F}_{\alpha, q}(E)\right\} .
\end{aligned}
$$

Interestingly, one can show that also the dependence of the Wigner function on the temperature can uniquely be expressed by the two parameters $\alpha_{\Gamma}=k_{\mathrm{B}} T / \Gamma$ and $\alpha_{\mathcal{E}}=k_{\mathrm{B}} T / \mathcal{E}$, introduced in this paper. A detailed discussion of this interesting temperature-dependence is beyond the scope of this paper and is postponed to a future publication.

Finally, we also express the noise (A5) in terms of Floquet scattering matrices and Fermi functions:

$$
\begin{aligned}
\mathcal{P}_{\alpha \beta}^{X Y}= & \frac{1}{h} \int d E \sum_{q, l, k=-\infty}^{\infty} x y_{q+l}\left\{\delta_{\alpha \beta} \delta_{q 0} \delta_{k 0} \mathcal{F}_{\alpha, l}(E)\left[\delta_{l 0}-\mathcal{F}_{\alpha,-l}\left(E_{l}\right)\right]-S_{\alpha \beta}^{*}\left(E, E_{q}\right) S_{\alpha \beta}\left(E, E_{q+k}\right)\right. \\
& \times \mathcal{F}_{\beta, l}\left(E_{q}\right)\left[\delta_{k l}-\mathcal{F}_{\beta, k-l}\left(E_{q+l}\right)\right]-S_{\beta \alpha}^{*}\left(E_{q+l}, E_{l}\right) S_{\beta \alpha}\left(E_{q+l}, E_{k}\right) \mathcal{F}_{\alpha, k}(E)\left[\delta_{l 0}-\mathcal{F}_{\alpha,-l}\left(E_{l}\right)\right] \\
& \left.+\sum_{\gamma \lambda} \sum_{n, m=-\infty}^{\infty} S_{\alpha \gamma}^{*}\left(E, E_{n}\right) S_{\alpha \lambda}\left(E, E_{m+k}\right) S_{\beta \lambda}^{*}\left(E_{q+l}, E_{m}\right) S_{\beta \gamma}\left(E_{q+l}, E_{n+l}\right) \mathcal{F}_{\gamma, l}\left(E_{n}\right)\left[\delta_{k 0}-\mathcal{F}_{\lambda, k}\left(E_{m}\right)\right]\right\} .
\end{aligned}
$$

In the equation above, we use a compact notation for the charge-current $\left(\mathcal{P}_{\alpha \beta}^{I I}\right)$, energy-current $\left(\mathcal{P}_{\alpha \beta}^{\mathcal{E} \mathcal{E}}\right)$ and mixed noise $\left(\mathcal{P}_{\alpha \beta}^{I \mathcal{E}}\right.$ and $\mathcal{P}_{\alpha \beta}^{\mathcal{E} I}$ ), which can be obtained by doing the replacements $x \rightarrow\{-e, E\}$ and $y_{q+l} \rightarrow\left\{-e, E_{q+l}\right\}$ for $X, Y \rightarrow\{I, \mathcal{E}\}$. Notice the notation used for distinguishing different types of noise where, for the sake of brevity, the superscript ' $\mathcal{E}$ ' refers to the energy current $I^{\mathcal{E}}$.

In order to relate these general expressions to the three setups discussed in the main text, the Floquet scattering matrices $S_{\alpha \beta}\left(E_{n}, E_{m}\right)$ have to be replaced by elements of the following matrices:

$$
S_{\mathrm{A}}\left(E_{n}, E_{m}\right)=\left(\begin{array}{cc}
\sqrt{1-D} S_{\text {cap }}\left(E_{n}, E_{m}\right) & \delta_{n m} \sqrt{D} \\
\sqrt{D} S_{\text {cap }}\left(E_{n}, E_{m}\right) & -\delta_{n m} \sqrt{1-D}
\end{array}\right)
$$

for the setup with the driven mesoscopic capacitor,

$$
S_{\mathrm{B}}\left(E_{n}, E_{m}\right)=\delta_{n m}\left(\begin{array}{cc}
\sqrt{1-D} & \sqrt{D} \\
\sqrt{D} & -\sqrt{1-D}
\end{array}\right)
$$


for the time-dependent bias voltage driving and

$$
S_{\mathrm{C}}\left(E_{n}, E_{m}\right)=\left(\begin{array}{cc}
\sqrt{1-D} e^{i E_{m} \tau_{\mathrm{g}} / \hbar} c_{\mathrm{g}, n-m} & \delta_{n m} \sqrt{D} \\
\sqrt{D} e^{i E_{m} \tau_{\mathrm{g}} / \hbar} c_{\mathrm{g}, n-m} & -\delta_{n m} \sqrt{1-D}
\end{array}\right)
$$

for the locally modulated edge state. Furthermore, in the cases A and C, namely, in the absence of a bias driving, only the amplitudes $c_{\mathrm{L}, 0}$ and $c_{\mathrm{R}, 0}$ contribute to the observables discussed in this section.

\section{APPENDIX C: ANALYTIC RESULTS FOR TIME-RESOLVED CURRENTS}

\section{Charge current}

The high-temperature $\left(\alpha_{\mathcal{E}} \gg 1\right)$ expression for the charge current $I_{\text {cap }}(t)$ for arbitrary $\alpha_{\Gamma}$ is obtained by performing explicitly the energy integration in Eq. (32), which yields

$$
I_{\text {cap }}(t)=\frac{e D}{2 \pi} \sum_{j=\mathrm{e}, \mathrm{h}}\left[\partial_{t} E_{\mathrm{res}}^{(j)}(t)\right]\left\{\left.\pi \partial_{E} \mathcal{A}_{\Gamma}(E)\right|_{E=E_{\mathrm{res}}^{(j)}(t)}+\frac{\beta}{2 \pi} \operatorname{Re} \Psi_{-}^{(1)}\left(\frac{\beta}{2 \pi}\left[E_{\mathrm{res}}^{(k)}(t)+i \Gamma\right]\right)\right\} .
$$

For the sake of notational brevity, in the equation above and henceforth, we use $\beta \equiv 1 /\left(k_{\mathrm{B}} T\right)$, and also employ the short notation $\partial_{x} \equiv \partial / \partial x$ for derivatives. The auxiliary functions are defined as

$$
\Psi_{ \pm}^{(n)}(z)=\psi^{(n)}(1 / 2+i z) \pm \psi^{(n)}(1 / 2-i z) \quad \text { and } \quad \mathcal{A}_{\Gamma}(E)=2 \operatorname{Re} f(E+i \Gamma)
$$

with $\psi^{(n)}(z)$ denoting the polygamma function of order $n$. Below we provide the explicit expression for the electron-emission contribution to the charge current,

$$
\frac{I_{\text {cap }}^{(\mathrm{e})}(\tilde{t})}{-e / \sigma}=\frac{D}{2 \pi \alpha_{\Gamma}}\left\{\pi \frac{1+\cosh \left(\tilde{t} / \alpha_{\Gamma}\right) \cos \left(1 / \alpha_{\Gamma}\right)}{\left[\cosh \left(\tilde{t} / \alpha_{\Gamma}\right)+\cos \left(1 / \alpha_{\Gamma}\right)\right]^{2}}-\frac{1}{2 \pi} \operatorname{Re} \Psi_{-}^{(1)}\left(\frac{1}{2 \pi \alpha_{\Gamma}}[\tilde{t}+i]\right)\right\},
$$

where the time $t$ is now scaled to the temporal width $\sigma$, that is, $\tilde{t}=t / \sigma$, and we have used Eq. (10) for the emission energy $E_{\text {res }}^{(\mathrm{e})}(t)$, which implies our choice of $t_{\text {cap }}^{(\mathrm{e})}(E=0)=0$. This equation is used to plot results shown in Fig. 2.

\section{Energy current}

Analogously as in the previous section, one can also derive the high-temperature $\left(\alpha_{\mathcal{E}} \gg 1\right)$ expression for the energy current $I_{\text {cap }}^{\mathcal{E}}(t)$ for arbitrary $\alpha_{\Gamma}$ by calculating in Eq. (36) the integrals with respect to energy,

$$
I_{\text {cap }}^{\mathcal{E}}(t)=\sum_{j=\mathrm{e}, \mathrm{h}}\left\{I_{\text {cap }, \mathrm{s}}^{\mathcal{E}(j)}(t)+I_{\text {cap ,as }}^{\mathcal{E}(j)}(t)\right\}
$$

where

$$
\begin{aligned}
I_{\text {cap }, \mathrm{s}}^{\mathcal{E}(j)}(t)= & -\frac{D}{2 \pi}\left[\partial_{t} E_{\mathrm{res}}^{(j)}(t)\right]\left\{E_{\mathrm{res}}^{(j)}(t)\left[\left.\pi \partial_{E} \mathcal{A}_{\Gamma}(E)\right|_{E=E_{\mathrm{res}}^{(j)}(t)}+\frac{\beta}{2 \pi} \operatorname{Re} \Psi_{-}^{(1)}\left(\frac{\beta}{2 \pi}\left[E_{\mathrm{res}}^{(j)}(t)+i \Gamma\right]\right)\right]\right. \\
& \left.+\Gamma\left[\left.\pi \partial_{E} \mathcal{B}_{\Gamma}(E)\right|_{E=E_{\mathrm{res}}^{(j)}(t)}-\frac{\beta}{2 \pi} \operatorname{Im} \Psi_{-}^{(1)}\left(\frac{\beta}{2 \pi}\left[E_{\mathrm{res}}^{(j)}(t)+i \Gamma\right]\right)\right]\right\},
\end{aligned}
$$

and

$$
\begin{aligned}
I_{\text {cap,as }}^{\mathcal{E}(j)}(t)= & \frac{D \hbar}{4 \pi}\left[\partial_{t} E_{\mathrm{res}}^{(j)}(t)\right]^{2}\left\{\frac{1}{\Gamma} E_{\mathrm{res}}^{(j)}(t)\left[\left.\pi \partial_{E}^{2} \mathcal{A}_{\Gamma}(E)\right|_{E=E_{\mathrm{res}}^{(j)}(t)}-\frac{\beta^{2}}{(2 \pi)^{2}} \operatorname{Im} \Psi_{+}^{(2)}\left(\frac{\beta}{2 \pi}\left[E_{\mathrm{res}}^{(j)}(t)+i \Gamma\right]\right)\right]\right. \\
& +\Gamma\left[\left.\pi \partial_{E}^{3} \mathcal{A}_{\Gamma}(E)\right|_{E=E_{\mathrm{res}}^{(j)}(t)}-\frac{\beta^{3}}{(2 \pi)^{3}} \operatorname{Re} \Psi_{-}^{(3)}\left(\frac{\beta}{2 \pi}\left[E_{\mathrm{res}}^{(j)}(t)+i \Gamma\right]\right)\right] \\
& \left.-E_{\mathrm{res}}^{(j)}(t)\left[\left.\pi \partial_{E}^{3} \mathcal{B}_{\Gamma}(E)\right|_{E=E_{\mathrm{res}}^{(j)}(t)}+\frac{\beta^{3}}{(2 \pi)^{3}} \operatorname{Im} \Psi_{-}^{(3)}\left(\frac{\beta}{2 \pi}\left[E_{\mathrm{res}}^{(j)}(t)+i \Gamma\right]\right)\right]\right\} .
\end{aligned}
$$

Recall that the auxiliary functions $\Psi_{\eta}^{(n)}(z)$ and $\mathcal{A}_{\Gamma}(E)$ are given by Eqs. (C2), while

$$
\mathcal{B}_{\Gamma}(E)=-2 \operatorname{Im} f(E+i \Gamma) .
$$


Furthermore, one can show that, for instance, the electron-emission contributions to the energy current take the explicit form [with $\tilde{t} \equiv t / \sigma]$

$$
\begin{aligned}
\frac{I_{\text {cap }, \mathrm{s}}^{\mathcal{E}(\mathrm{e})}(\tilde{t})}{\mathcal{E} / \sigma}= & \frac{D}{2 \pi} \cdot \frac{\Gamma}{\mathcal{E}}\left\{\frac{\tilde{t}}{\alpha_{\Gamma}}\left[\pi \frac{1+\cosh \left(\tilde{t} / \alpha_{\Gamma}\right) \cos \left(1 / \alpha_{\Gamma}\right)}{\left[\cosh \left(\tilde{t} / \alpha_{\Gamma}\right)+\cos \left(1 / \alpha_{\Gamma}\right)\right]^{2}}-\frac{1}{2 \pi} \operatorname{Re} \Psi_{-}^{(1)}\left(\frac{1}{2 \pi \alpha_{\Gamma}}[\tilde{t}+i]\right)\right]\right. \\
& \left.+\frac{1}{\alpha_{\Gamma}}\left[\pi \frac{\sinh \left(\tilde{t} / \alpha_{\Gamma}\right) \sin \left(1 / \alpha_{\Gamma}\right)}{\left[\cosh \left(\tilde{t} / \alpha_{\Gamma}\right)+\cos \left(1 / \alpha_{\Gamma}\right)\right]^{2}}+\frac{1}{2 \pi} \operatorname{Im} \Psi_{-}^{(1)}\left(\frac{1}{2 \pi \alpha_{\Gamma}}[\tilde{t}+i]\right)\right]\right\}
\end{aligned}
$$

and

$$
\begin{aligned}
\frac{I_{\text {cap, as }}^{\mathcal{E}(\mathrm{e})}(\tilde{t})}{\mathcal{E} / \sigma}= & \frac{D}{2 \pi}\left\{\frac{\tilde{t}}{\alpha_{\Gamma}^{2}}\left[\pi \sinh \left(\tilde{t} / \alpha_{\Gamma}\right) \frac{2-\cos ^{2}\left(1 / \alpha_{\Gamma}\right)+\cosh \left(\tilde{t} / \alpha_{\Gamma}\right) \cos \left(1 / \alpha_{\Gamma}\right)}{\left[\cosh \left(\tilde{t} / \alpha_{\Gamma}\right)+\cos \left(1 / \alpha_{\Gamma}\right)\right]^{3}}-\frac{1}{(2 \pi)^{2}} \operatorname{Im} \Psi_{+}^{(2)}\left(\frac{1}{2 \pi \alpha_{\Gamma}}[\tilde{t}+i]\right)\right]\right. \\
& +\frac{1}{\alpha_{\Gamma}^{3}}\left[\pi \mathcal{C}_{\mathcal{A}}\left(\tilde{t}, \alpha_{\Gamma}\right)-\frac{1}{(2 \pi)^{3}} \operatorname{Re} \Psi_{-}^{(3)}\left(\frac{1}{2 \pi \alpha_{\Gamma}}[\tilde{t}+i]\right)\right] \\
& \left.-\frac{\tilde{t}}{\alpha_{\Gamma}^{3}}\left[\pi \mathcal{C}_{\mathcal{B}}\left(\tilde{t}, \alpha_{\Gamma}\right)+\frac{1}{(2 \pi)^{3}} \operatorname{Im} \Psi_{-}^{(3)}\left(\frac{1}{2 \pi \alpha_{\Gamma}}[\tilde{t}+i]\right)\right]\right\}
\end{aligned}
$$

where the auxiliary functions $\mathcal{C}_{\mathcal{A}}\left(\tilde{t}, \alpha_{\Gamma}\right)$ and $\mathcal{C}_{\mathcal{B}}\left(\tilde{t}, \alpha_{\Gamma}\right)$ are given by

$$
\begin{gathered}
\mathcal{C}_{\mathcal{A}}\left(\tilde{t}, \alpha_{\Gamma}\right)=\frac{1}{\left[\cosh \left(\tilde{t} / \alpha_{\Gamma}\right)+\cos \left(1 / \alpha_{\Gamma}\right)\right]^{4}}\left\{3-\cosh \left(2 \tilde{t} / \alpha_{\Gamma}\right)+2 \sinh ^{2}\left(\tilde{t} / \alpha_{\Gamma}\right) \cos \left(2 / \alpha_{\Gamma}\right)\right. \\
\left.-\frac{1}{2} \cosh \left(\tilde{t} / \alpha_{\Gamma}\right) \cos \left(1 / \alpha_{\Gamma}\right)\left[-6+\cos \left(2 / \alpha_{\Gamma}\right)+\cosh \left(2 \tilde{t} / \alpha_{\Gamma}\right)\right]\right\}, \\
\mathcal{C}_{\mathcal{B}}\left(\tilde{t}, \alpha_{\Gamma}\right)=-\sinh \left(\tilde{t} / \alpha_{\Gamma}\right) \sin \left(1 / \alpha_{\Gamma}\right) \frac{\cos \left(2 / \alpha_{\Gamma}\right)+\cosh \left(2 \tilde{t} / \alpha_{\Gamma}\right)-8 \cosh \left(\tilde{t} / \alpha_{\Gamma}\right) \cos \left(1 / \alpha_{\Gamma}\right)-10}{2\left[\cosh \left(\tilde{t} / \alpha_{\Gamma}\right)+\cos \left(1 / \alpha_{\Gamma}\right)\right]^{4}} .
\end{gathered}
$$

These equations are used to plot results shown in Fig. 3 as well as Fig. 8.

\section{APPENDIX D: TEMPERATURE DEPENDENCE OF THE TIME-RESOLVED ENERGY CURRENT}

In this brief Appendix, we separately present plots of the antisymmetric and symmetric contribution of the timeresolved energy current of the capacitor, $I_{\text {cap }, \mathrm{S}}^{\mathcal{E}}(t)$ and $I_{\text {cap,as }}^{\mathcal{E}}(t)$, in the high-temperature limit, as given in Eq. (36) and explicitly evaluated in Eqs. (C5) and (C6). For simplicity, we only show the electronic part, cf. Eqs. (C8) and (C9); the hole emission yields equivalent results shifted by one half of the period. Panels (a) and (b) in the left column of Fig. 8 show the behavior for fixed $\alpha_{\mathcal{E}}=10$ for different values of $\alpha_{\Gamma}$. We see that with increasing $\alpha_{\Gamma}$, an antisymmetric contribution arises, which is fully absent in the zero-temperature limit. For $\alpha_{\Gamma} \simeq 1$, the absolute value of the maximum/minimum values of this contribution stop increasing. Subsequently, merely a broadening, going along with a shift of the time at which the maximum/minimum occurs, $t_{\max } \approx \pm \sigma \alpha_{\Gamma}$, can be observed.

The symmetric component, $I_{\text {cap, }}^{\mathcal{E}}(t)$, shown in panel (b) has a very different behavior as function of $\alpha_{\Gamma}$. The peak at $t=0$, which is observed at zero temperature, splits into two peaks, again with maxima occurring at approximately $t_{\max } \approx \pm \alpha_{\Gamma} \sigma$, and with the temperature broadening. The integral over the whole curve remains constant with changing $\alpha_{\Gamma}$.

Interestingly, the influence of $\alpha_{\mathcal{E}}$, shown in panels (c) and (d) in the right column of Fig. 8 barely results in an overall increase of the weight of the antisymmetric part of the function $I_{\text {cap }}^{\mathcal{E}}(t)$. The symmetric contribution is completely independent of the factor $\alpha_{\mathcal{E}}$.
This behavior reflects that explicitly presented in Eq. (38) for $\alpha_{\Gamma} \gg 1$. Note, however, that the plots shown in this Appendix are not restricted to the regime of $\alpha_{\Gamma} \gg 1$.

\section{APPENDIX E: ANALYTIC RESULTS FOR SPECTRAL CURRENTS}

1. Time-dependently driven mesoscopic capacitor (setup A) and locally modulated edge state (setup C)

In the case of the time-dependently driven mesoscopic capacitor and the locally modulated edge state, one employs Eq. (4) for $\left|S\left(E, E_{n}\right)\right|^{2}$ and the equivalent expression from Eq. (19) [in combination with Eq. (23)], respectively, so that Eq. (39) reads

$i_{\text {cap } / \mathrm{loc}}(E)=(2 \Omega \sigma)^{2} D \sum_{n=0}^{\infty} e^{-2 n \Omega \sigma}\left[f\left(E_{n}\right)+f\left(E_{-n}\right)-2 f(E)\right]$.

Next, in the limit $\alpha_{\mathcal{E}} \gg 1$ considered here, ${ }^{7}$ one can convert the sum in Eq. (E1) into an integral over $\omega=n \hbar \Omega /\left(k_{\mathrm{B}} T\right)$,

$$
\begin{aligned}
i_{\text {cap } / \mathrm{loc}}(E)= & 2 \Omega \sigma D \alpha_{\mathcal{E}} \int_{0}^{\infty} d \omega e^{-\omega \alpha_{\mathcal{E}}}\left[f\left(E+\omega k_{\mathrm{B}} T\right)\right. \\
& \left.+f\left(E-\omega k_{\mathrm{B}} T\right)-2 f(E)\right] .
\end{aligned}
$$

\footnotetext{
${ }^{7}$ Note that, strictly speaking, the condition $k_{\mathrm{B}} T \gg \hbar \Omega$, which is weaker than $\alpha_{\mathcal{E}} \gg 1$, is sufficient for the transformation from the sum into an integral.
} 

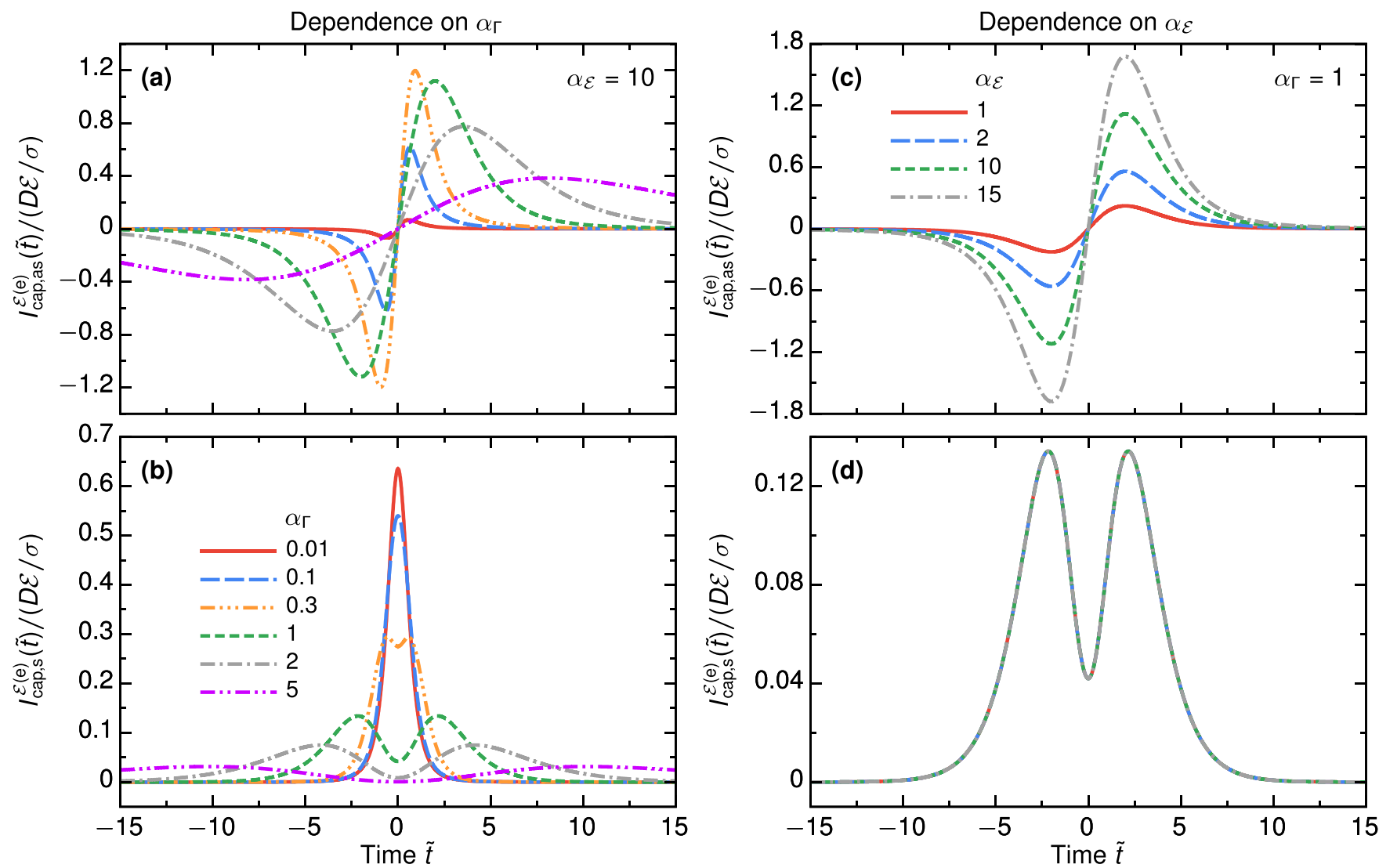

FIG. 8. Electron-contribution to the time-resolved energy current emitted from the driven mesoscopic capacitor (setup A) as a function of the dimensionless time $\tilde{t}=t / \sigma$. Here, the high-temperature limit, $\alpha_{\mathcal{E}}=k_{\mathrm{B}} T / \mathcal{E}>1$ is considered for different values of $\alpha_{\mathcal{E}}$ and $\alpha_{\Gamma}=k_{\mathrm{B}} T / \Gamma$. We present the antisymmetric $I_{\text {cap,as }}^{\mathcal{E}}(t)$ and symmetric $I_{\text {cap }, \mathrm{s}}^{\mathcal{E}}(t)$ contributions to Eq. (36) separately.

From there we obtain with the help of the definition of the (Gauss) hypergeometric function ${ }_{2} F_{1}(a, b, c ; z)$ [78],

$$
\begin{aligned}
& i_{\text {cap } / \mathrm{loc}}(\widetilde{E}) \\
& \quad=2 \Omega \sigma D\left\{\tanh \left(\frac{\widetilde{E}}{2 \alpha_{\mathcal{E}}}\right)+{ }_{2} \widetilde{F}_{1}\left(\alpha_{\mathcal{E}} ; \mathrm{e}^{-\widetilde{E} / \alpha_{\mathcal{E}}}\right)-{ }_{2} \widetilde{F}_{1}\left(\alpha_{\mathcal{E}} ; \mathrm{e}^{\widetilde{E} / \alpha_{\mathcal{E}}}\right)\right\},
\end{aligned}
$$

where the dimensionless energy $\widetilde{E}=E / \mathcal{E}$ has been introduced, and

$$
{ }_{2} \widetilde{F}_{1}\left(\alpha_{\mathcal{E}} ; z\right)=\frac{\alpha_{\mathcal{E}}}{1+\alpha_{\mathcal{E}}} z_{2} F_{1}\left(1,1+\alpha_{\mathcal{E}}, 2+\alpha_{\mathcal{E}} ;-z\right) .
$$

Results corresponding to Eq. (E3) are plotted in Fig. 4(a).

\section{Lorentzian bias driving (setup B)}

In order to evaluate the sum in Eq. (39) for the spectral current in the case of the Lorentzian bias driving, let us first replace $\left|S\left(E, E_{n}\right)\right|^{2}$ by $\left|c_{\mathrm{L}, n}\right|^{2}$ given by Eq. (18) and use that $\bar{V}_{\mathrm{b}}=\hbar \Omega /(-e)$,

$$
i_{\mathrm{lev}}(E)=(2 \Omega \sigma)^{2} D \sum_{n=0}^{\infty} e^{-2 n \Omega \sigma}\left[f\left(E_{n}\right)-f(E)\right] .
$$

Then, as discussed in the previous subsection, one can write

$$
i_{\mathrm{lev}}(E)=2 \Omega \sigma D \alpha_{\mathcal{E}} \int_{0}^{\infty} d \omega e^{-\omega \alpha_{\mathcal{E}}}\left[f\left(E+\omega k_{\mathrm{B}} T\right)-f(E)\right]
$$

With the help of the hypergeometric function ${ }_{2} F_{1}(a, b, c ; z)$, the equation above can be shown to have the following form:

$$
i_{\mathrm{lev}}(\widetilde{E})=2 \sigma \Omega D\left[\frac{1}{1+\mathrm{e}^{-\widetilde{E} / \alpha_{\mathcal{E}}}}-{ }_{2} \widetilde{F}_{1}\left(\alpha_{\mathcal{E}} ; \mathrm{e}^{\widetilde{E} / \alpha_{\mathcal{E}}}\right)\right] .
$$

Equation (E7) has been used to plot results shown in Fig. 4(b).

\section{APPENDIX F: DERIVATION OF THE ENERGY-CURRENT NOISE}

In this Appendix, we derive the general expressions for the noises in setup A, based on a mesoscopic capacitor. Using Eqs. (B11) and (B12) in the absence of a bias driving 
$\mathcal{F}_{\alpha, q}(E)=\delta_{q, 0} f_{\alpha}(E)$, we find

$$
\begin{aligned}
\mathcal{P}_{\text {cap }}^{X Y}= & \frac{1}{h} \sum_{n=-\infty}^{\infty}\left\{D(1-D) \int d E x y\left|S_{\text {cap }}\left(E, E_{-n}\right)\right|^{2} A_{\mathrm{LR}}^{n}(E)+D^{2}\left[\delta_{n 0} \int d E x y F_{\mathrm{RR}}^{0,0}(E)\right.\right. \\
& \left.\left.+\sum_{k, l=-\infty}^{\infty} \int d E x y_{n} S_{\text {cap }}^{*}\left(E, E_{-l}\right) S_{\text {cap }}\left(E_{n}, E_{-l}\right) S_{\text {cap }}^{*}\left(E_{n}, E_{-k}\right) S_{\text {cap }}\left(E, E_{-k}\right) F_{\mathrm{LL}}^{l, k}(E)\right]\right\},
\end{aligned}
$$

where we have introduced two auxiliary functions $F_{\alpha \beta}^{n, m}(E)$ and $A_{\alpha \beta}^{q}(E)$ defined as

$$
F_{\alpha \beta}^{n, m}(E) \equiv f_{\alpha}\left(E_{-n}\right)\left[1-f_{\beta}\left(E_{-m}\right)\right] \quad \text { and } \quad A_{\alpha \beta}^{q}(E) \equiv F_{\alpha \beta}^{q, 0}(E)+F_{\beta \alpha}^{0, q}(E) .
$$

First of all, it can be seen that formally Eq. (F1) consists of two types of terms-the first $\propto D(1-D)$ and the second $\propto D^{2}-$ which have a clear physical interpretation [40,53]. Specifically, the term $\propto D(1-D)$ describes correlations generated by the exchange of particles between two different reservoirs, and hence, it is often referred to as the transport part of the noise. Conversely, the term $\propto D^{2}$, the so-called interference part of the noise, represents correlations due to the exchange of particles between states with different energies in the same reservoir.

Unlike for the charge-current noise, see Sec. V A, where the dependence of the scattering matrix $S_{\text {cap }}\left(E_{n}, E_{m}\right)$ on energy effectively did not play a role, for the energy-current noise this dependence has to be carefully taken into account. In particular, from Eq. (F1), we derive

$$
\begin{aligned}
\mathcal{P}_{\text {cap }}^{\mathcal{E E}}= & \frac{D(1-D)}{3 h} \sum_{n=-\infty}^{\infty}\left|S_{n}\right|^{2} \operatorname{coth}\left(\frac{n \hbar \Omega}{2 k_{\mathrm{B}} T}\right)\left[(n \hbar \Omega)^{3}+n \hbar \Omega\left(\pi k_{\mathrm{B}} T\right)^{2}\right] \\
& +\frac{D^{2}}{h}\left\{\frac{2 \pi^{2}}{3}\left(k_{\mathrm{B}} T\right)^{3}+\sum_{n>0}^{\infty} \operatorname{coth}\left(\frac{n \hbar \Omega}{2 k_{\mathrm{B}} T}\right) \int d E\left|\Lambda_{n}(E)\right|^{2}\left[f(E)-f\left(E_{n}\right)\right]\right\},
\end{aligned}
$$

where

$$
\Lambda_{n}(E)=\sum_{k=-\infty}^{\infty} k \hbar \Omega S_{\text {cap }}^{*}\left(E_{k}, E\right) S_{\text {cap }}\left(E_{k}, E_{n}\right) .
$$

Comparing the equation above to the analogous equation for the Lorentzian bias driving (cf. Eqs. (13) and (15) of Ref. [40]), it can be noticed that apart from the lack of a bias voltage applied to contacts, one finds in Eq. (F3) an additional term —originating from the interference part of Eq. (F1) — which arises due to the energy dependence of the scattering matrix. Replacing the scattering matrices in Eq. (F4) by their explicit form (4) [recall that $\left|S_{n}\right|^{2}$ in Eq. (F3) is given by Eq. (40)], and finding that

$$
\left|\Lambda_{n}(E)\right|^{2}=2(\hbar \Omega)^{2} e^{-2 n \Omega \sigma}\{1-\cos [n \Omega \Delta t(E)]\},
$$

with $\Delta t(E) \equiv t^{(\mathrm{e})}(E)-t^{(\mathrm{h})}(E)$, we eventually obtain

$$
\begin{aligned}
\mathcal{P}_{\text {cap }}^{\mathcal{E E}}= & \frac{2 \pi^{2}}{3 h} D\left(k_{\mathrm{B}} T\right)^{3}+\frac{2 D^{2}}{h}(\hbar \Omega)^{2} \sum_{n>0}^{\infty} e^{-2 n \Omega \sigma} n \hbar \Omega \operatorname{coth}\left(\frac{n \hbar \Omega}{2 k_{\mathrm{B}} T}\right) \\
& +\frac{D(1-D)}{3 h}(2 \Omega \sigma)^{2} \sum_{n=-\infty}^{\prime} e^{-2|n| \Omega \sigma} \operatorname{coth}\left(\frac{n \hbar \Omega}{2 k_{\mathrm{B}} T}\right)\left[(n \hbar \Omega)^{3}+n \hbar \Omega\left(\pi k_{\mathrm{B}} T\right)^{2}\right]+\left(\mathcal{P}_{\text {cap }}^{\mathcal{E \mathcal { E }}}\right)^{\prime} .
\end{aligned}
$$

Here, the additional term $\left(\mathcal{P}_{\text {cap }}^{\mathcal{E E}}\right)^{\prime}$ is given by

$$
\left(\mathcal{P}_{\text {cap }}^{\mathcal{E \mathcal { E }}}\right)^{\prime} \equiv-\frac{2 D^{2}}{h}(\hbar \Omega)^{2} \sum_{n>0}^{\infty}(-1)^{n} e^{-2 n \Omega \sigma} \operatorname{coth}\left(\frac{n \hbar \Omega}{2 k_{\mathrm{B}} T}\right) \mathcal{J}_{n},
$$

with

$$
\mathcal{J}_{n}=\int d E \cos \left[2 n \Omega t^{(\mathrm{e})}(E)\right]\left\{f(E)-f\left(E_{n}\right)\right\}=\pi k_{\mathrm{B}} T \sin \left(2 n^{2} \frac{\hbar \Omega}{e \delta U}\right) \sinh ^{-1}\left(2 \pi n \frac{k_{\mathrm{B}} T}{e \delta U}\right) .
$$

Here, we have used that $\cos [n \Omega \Delta t(E)]=(-1)^{n} \cos \left[2 n \Omega t^{(\mathrm{e})}(E)\right]$, from Eq. (5). Importantly, the term $\left(\mathcal{P}_{\text {cap }}^{\mathcal{E E}}\right)^{\prime}$ can be conveniently estimated for the parameter regime considered in this paper by invoking the conditions (2) and (3), i.e., $\Omega \sigma \ll 1$ and $k_{\mathrm{B}} T /|e \delta U| \ll 1$, and noting that the energy of the Floquet quantum, $\hbar \Omega$, is in fact the smallest energy scale under the adiabatic-driving condition, meaning that $\hbar \Omega /\left(k_{\mathrm{B}} T\right) \ll 1$. In order to analyze $\left(\mathcal{P}_{\text {cap }}^{\mathcal{E E}}\right)^{\prime}$, let us insert Eq. (F8) into Eq. (F7), which 
yields

$$
\frac{\left(\mathcal{P}_{\text {cap }}^{\mathcal{E}}\right)^{\prime}}{\mathcal{E}^{2} / \mathcal{T}}=-4 D^{2} \sigma \Omega \alpha_{\mathcal{E}} \sum_{n>0}^{\infty}(-1)^{n} a_{n} \quad \text { with } \quad a_{n}=\pi e^{-2 n \Omega \sigma} \operatorname{coth}\left(\frac{n \hbar \Omega}{2 k_{\mathrm{B}} T}\right) \sin \left(2 n^{2} \frac{\hbar \Omega}{k_{\mathrm{B}} T} \cdot \frac{k_{\mathrm{B}} T}{e \delta U}\right) \sinh ^{-1}\left(2 \pi n \frac{k_{\mathrm{B}} T}{e \delta U}\right),
$$

Then, the key task is reduced to the calculation of the series $\sum_{n>0}^{\infty}(-1)^{n} a_{n}$. This series is convergent (as it is absolutely converging, which can be shown from the comparison test). Furthermore, in the parameter range mentioned above $a_{n}$ and $a_{n+1}$ differ very little for all $n$. We can therefore approximate this series as follows:

$$
\sum_{n>0}^{\infty}(-1)^{n} a_{n} \approx-\left.\sum_{k=0}^{\infty} \frac{d a_{n}}{d n}\right|_{n=2 k+1} \approx-\frac{1}{2} \int_{0}^{\infty} d n \frac{d a_{n}}{d n}=1,
$$

so that we obtain

$$
\frac{\left(\mathcal{P}_{\text {cap }}^{\mathcal{E E}}\right)^{\prime}}{\mathcal{E}^{2} / \mathcal{T}}=-4 D^{2} \sigma \Omega \alpha_{\mathcal{E}}
$$

Consequently, we can conclude that under the conditions considered in this paper, $\left(\mathcal{P}_{\text {cap }}^{\mathcal{E E}}\right)^{\prime}$ is negligibly small as compared to the remaining terms in Eq. (F6), see also Eqs. (45) for explicit expressions.

[1] E. Bocquillon, V. Freulon, F. D. Parmentier, J.-M. Berroir, B. Plaçais, C. Wahl, J. Rech, T. Jonckheere, T. Martin, C. Grenier, D. Ferraro, P. Degiovanni, and G. Fève, Electron quantum optics in ballistic chiral conductors, Ann. Phys. 526, 1 (2014).

[2] C. Bäuerle, D. C. Glattli, T. Meunier, F. Portier, P. Roche, P. Roulleau, S. Takada, and X. Waintal, Coherent control of single electrons: a review of current progress, Rep. Prog. Phys. 81, 056503 (2018).

[3] M. D. Blumenthal, B. Kaestner, L. Li, S. Giblin, T. J. B. M. Janssen, M. Pepper, D. Anderson, G. Jones, and D. A. Ritchie, Gigahertz quantized charge pumping, Nat. Phys. 3, 343 (2007).

[4] G. Fève, A. Mahé, J.-M. Berroir, T. Kontos, B. Plaçais, D. C. Glattli, A. Cavanna, B. Etienne, and Y. Jin, An On-Demand Coherent Single-Electron Source, Science 316, 1169 (2007).

[5] R. P. G. McNeil, M. Kataoka, C. J. B. Ford, C. H. W. Barnes, D. Anderson, G. A. C. Jones, I. Farrer, and D. A. Ritchie, Ondemand single-electron transfer between distant quantum dots, Nature (London) 477, 439 (2011).

[6] S. Hermelin, S. Takada, M. Yamamoto, S. Tarucha, A. D. Wieck, L. Saminadayar, C. Bäuerle, and T. Meunier, Electrons surfing on a sound wave as a platform for quantum optics with flying electrons, Nature (London) 477, 435 (2011).

[7] J. Dubois, T. Jullien, F. Portier, P. Roche, A. Cavanna, Y. Jin, W. Wegscheider, P. Roulleau, and D. C. Glattli, Minimalexcitation states for electron quantum optics using levitons, Nature (London) 502, 659 (2013).

[8] J. D. Fletcher, P. See, H. Howe, M. Pepper, S. P. Giblin, J. P. Griffiths, G. A. C. Jones, I. Farrer, D. A. Ritchie, T. J. B. M. Janssen, and M. Kataoka, Clock-Controlled Emission of SingleElectron Wave Packets in a Solid-State Circuit, Phys. Rev. Lett. 111, 216807 (2013).

[9] N. Ubbelohde, F. Hohls, V. Kashcheyevs, T. Wagner, L. Fricke, B. Kästner, K. Pierz, H. W. Schumacher, and R. J. Haug, Partitioning of on-demand electron pairs, Nat. Nanotechnol. 10, 46 (2014).

[10] D. M. T. van Zanten, D. M. Basko, I. M. Khaymovich, J. P. Pekola, H. Courtois, and C. B. Winkelmann, Single Quantum Level Electron Turnstile, Phys. Rev. Lett. 116, 166801 (2016).
[11] G. Haack, M. Moskalets, J. Splettstoesser, and M. Büttiker, Coherence of single-electron sources from Mach-Zehnder interferometry, Phys. Rev. B 84, 081303(R) (2011).

[12] S. Juergens, J. Splettstoesser, and M. Moskalets, Single-particle interference versus two-particle collisions, Europhys. Lett. 96, 37011 (2011).

[13] P. P. Hofer and C. Flindt, Mach-Zehnder interferometry with periodic voltage pulses, Phys. Rev. B 90, 235416 (2014).

[14] G. Rosselló, F. Battista, M. Moskalets, and J. Splettstoesser, Interference and multiparticle effects in a Mach-Zehnder interferometer with single-particle sources, Phys. Rev. B 91, 115438 (2015).

[15] D. Dasenbrook and C. Flindt, Dynamical generation and detection of entanglement in neutral leviton pairs, Phys. Rev. B 92, 161412(R) (2015).

[16] C. Grenier, R. Hervé, E. Bocquillon, F. D. Parmentier, B. Plaçais, J. M. Berroir, G. Fève, and P. Degiovanni, Singleelectron quantum tomography in quantum Hall edge channels, New J. Phys. 13, 093007 (2011).

[17] E. Bocquillon, F. D. Parmentier, C. Grenier, J.-M. Berroir, P. Degiovanni, D. C. Glattli, B. Plaçais, A. Cavanna, Y. Jin, and G. Fève, Electron Quantum Optics: Partitioning Electrons One by One, Phys. Rev. Lett. 108, 196803 (2012).

[18] E. Bocquillon, V. Freulon, J.-M. Berroir, P. Degiovanni, B. Plaçais, A. Cavanna, Y. Jin, and G. Fève, Separation of neutral and charge modes in one-dimensional chiral edge channels, Nat. Commun. 4, 1839 (2013).

[19] T. Jullien, P. Roulleau, B. Roche, A. Cavanna, Y. Jin, and D. C. Glattli, Quantum tomography of an electron, Nature (London) 514, 603 (2014).

[20] É. Thibierge, D. Ferraro, B. Roussel, C. Cabart, A. Marguerite, G. Fève, and P. Degiovanni, Two-electron coherence and its measurement in electron quantum optics, Phys. Rev. B 93, 081302(R) (2016).

[21] S. Ol'khovskaya, J. Splettstoesser, M. Moskalets, and M. Büttiker, Shot Noise of a Mesoscopic Two-Particle Collider, Phys. Rev. Lett. 101, 166802 (2008). 
[22] M. Moskalets and M. Büttiker, Spectroscopy of electron flows with single- and two-particle emitters, Phys. Rev. B 83, 035316 (2011).

[23] T. Jonckheere, J. Rech, C. Wahl, and T. Martin, Electron and hole Hong-Ou-Mandel interferometry, Phys. Rev. B 86, 125425 (2012).

[24] E. Bocquillon, V. Freulon, J.-M. Berroir, P. Degiovanni, B. Plaçais, A. Cavanna, Y. Jin, and G. Fève, Coherence and Indistinguishability of Single Electrons Emitted by Independent Sources, Science 339, 1054 (2013).

[25] C. Wahl, J. Rech, T. Jonckheere, and T. Martin, Interactions and Charge Fractionalization in an Electronic HongOu-Mandel Interferometer, Phys. Rev. Lett. 112, 046802 (2014).

[26] V. Freulon, A. Marguerite, J.-M. Berroir, B. Plaçais, A. Cavanna, Y. Jin, and G. Fève, Hong-Ou-Mandel experiment for temporal investigation of single-electron fractionalization, Nat. Commun. 6, 6854 (2015).

[27] A. Marguerite, C. Cabart, C. Wahl, B. Roussel, V. Freulon, D. Ferraro, Ch. Grenier, J.-M. Berroir, B. Plaçais, T. Jonckheere, J. Rech, T. Martin, P. Degiovanni, A. Cavanna, Y. Jin, and G. Fève, Decoherence and relaxation of a single electron in a onedimensional conductor, Phys. Rev. B 94, 115311 (2016).

[28] A. Marguerite, B. Roussel, R. Bisognin, C. Cabart, M. Kumar, J.-M. Berroir, E. Bocquillon, B. Plaçais, A. Cavanna, U. Gennser, Y. Jin, P. Degiovanni, and G. Fève, Extracting single electron wavefunctions from a quantum electrical current, arXiv:1710.11181.

[29] E. Iyoda, T. Kato, K. Koshino, and T. Martin, Dephasing in single-electron generation due to environmental noise probed by Hong-Ou-Mandel interferometry, Phys. Rev. B 89, 205318 (2014).

[30] C. Cabart, B. Roussel, G. Fève, and P. Degiovanni, Taming electronic decoherence in one-dimensional chiral ballistic quantum conductors, Phys. Rev. B 98, 155302 (2018).

[31] J. Gabelli and B. Reulet, Shaping a time-dependent excitation to minimize the shot noise in a tunnel junction, Phys. Rev. B 87, 075403 (2013).

[32] V. Kashcheyevs and P. Samuelsson, Classical-to-quantum crossover in electron on-demand emission, Phys. Rev. B 95, 245424 (2017).

[33] J. Splettstoesser, M. Moskalets, and M. Büttiker, Two-Particle Nonlocal Aharonov-Bohm Effect from Two Single-Particle Emitters, Phys. Rev. Lett. 103, 076804 (2009).

[34] Y. Sherkunov, N. d'Ambrumenil, P. Samuelsson, and M. Büttiker, Optimal pumping of orbital entanglement with singleparticle emitters, Phys. Rev. B 85, 081108(R) (2012).

[35] A. A. Vyshnevyy, G. B. Lesovik, T. Jonckheere, and T. Martin, Setup of three Mach-Zehnder interferometers for production and observation of Greenberger-Horne-Zeilinger entanglement of electrons, Phys. Rev. B 87, 165417 (2013).

[36] P. P. Hofer and M. Büttiker, Emission of time-bin entangled particles into helical edge states, Phys. Rev. B 88, 241308(R) (2013).

[37] A. Inhofer and D. Bercioux, Proposal for an on-demand source of polarized electrons into the edges of a topological insulator, Phys. Rev. B 88, 235412 (2013).

[38] M. Moskalets and M. Büttiker, Heat production and current noise for single- and double-cavity quantum capacitors, Phys. Rev. B 80, 081302(R) (2009).
[39] F. Battista, M. Moskalets, M. Albert, and P. Samuelsson, Quantum Heat Fluctuations of Single-Particle Sources, Phys. Rev. Lett. 110, 126602 (2013).

[40] F. Battista, F. Haupt, and J. Splettstoesser, Energy and power fluctuations in ac-driven coherent conductors, Phys. Rev. B 90, 085418 (2014).

[41] M. Moskalets, Floquet Scattering Matrix Theory of Heat Fluctuations in Dynamical Quantum Conductors, Phys. Rev. Lett. 112, 206801 (2014)

[42] F. Battista, F. Haupt, and J. Splettstoesser, Correlations between charge and energy current in ac-driven coherent conductors, J. Phys.: Conf. Ser. 568, 052008 (2014).

[43] M. Moskalets and G. Haack, Heat and charge transport measurements to access single-electron quantum characteristics, Phys. Status Solidi B 254, 1600616 (2017).

[44] L. Vannucci, F. Ronetti, J. Rech, D. Ferraro, T. Jonckheere, T. Martin, and M. Sassetti, Minimal excitation states for heat transport in driven quantum Hall systems, Phys. Rev. B 95, 245415 (2017).

[45] N. Dashti, M. Misiorny, P. Samuelsson, and J. Splettstoesser, Probing Charge- and Heat-Current Noise by FrequencyDependent Fluctuations in Temperature and Potential, Phys. Rev. Appl. 10, 024007 (2018).

[46] F. Ronetti, L. Vannucci, D. Ferraro, T. Jonckheere, J. Rech, T. Martin, and M. Sassetti, Hong-Ou-Mandel heat noise in the quantum Hall regime, Phys. Rev. B 99, 205406 (2019).

[47] A. Prêtre, H. Thomas, and M. Büttiker, Dynamic admittance of mesoscopic conductors: Discrete-potential model, Phys. Rev. B 54, 8130 (1996).

[48] M. Büttiker, H. Thomas, and A. Prêtre, Mesoscopic capacitors, Phys. Lett. A 180, 364 (1993).

[49] L. S. Levitov, H. Lee, and G. B. Lesovik, Electron counting statistics and coherent states of electric current, J. Math. Phys. 37, 4845 (1996).

[50] J. Keeling, I. Klich, and L. S. Levitov, Minimal Excitation States of Electrons in One-Dimensional Wires, Phys. Rev. Lett. 97, 116403 (2006).

[51] J. Keeling, A. V. Shytov, and L. S. Levitov, Coherent Particle Transfer in an On-Demand Single-Electron Source, Phys. Rev. Lett. 101, 196404 (2008).

[52] Y. Sherkunov, J. Zhang, N. d'Ambrumenil, and B. Muzykantskii, Optimal electron entangler and single-electron source at low temperatures, Phys. Rev. B 80, 041313(R) (2009).

[53] J. Dubois, T. Jullien, C. Grenier, P. Degiovanni, P. Roulleau, and D. C. Glattli, Integer and fractional charge Lorentzian voltage pulses analyzed in the framework of photon-assisted shot noise, Phys. Rev. B 88, 085301 (2013).

[54] M. Misiorny, G. Fève, and J. Splettstoesser, Shaping charge excitations in chiral edge states with a time-dependent gate voltage, Phys. Rev. B 97, 075426 (2018).

[55] M. V. Moskalets, Scattering Matrix Approach to Non-Stationary Quantum Transport (Imperial College Press, London, 2011).

[56] M. Moskalets, P. Samuelsson, and M. Büttiker, Quantized Dynamics of a Coherent Capacitor, Phys. Rev. Lett. 100, 086601 (2008).

[57] J. Splettstoesser, S. Ol'khovskaya, M. Moskalets, and M. Büttiker, Electron counting with a two-particle emitter, Phys. Rev. B 78, 205110 (2008). 
[58] J. Gabelli, G. Fève, J.-M. Berroir, B. Plaçais, A. Cavanna, B. Etienne, Y. Jin, and D. C. Glattli, Violation of Kirchhoff's Laws for a Coherent $R C$ Circuit, Science 313, 499 (2006).

[59] M. Moskalets and G. Haack, Single-electron coherence: Finite temperature versus pure dephasing, Physica E 75, 358 (2016).

[60] M. Moskalets, Single-particle emission at finite temperatures, Low Temp. Phys. 43, 865 (2017).

[61] M. Moskalets, Single-particle shot noise at nonzero temperature, Phys. Rev. B 96, 165423 (2017).

[62] C. Altimiras, H. Le Sueur, U. Gennser, A. Cavanna, D. Mailly, and F. Pierre, Non-equilibrium edge-channel spectroscopy in the integer quantum Hall regime, Nat. Phys. 6, 34 (2009).

[63] F. Battista and P. Samuelsson, Spectral distribution and wave function of electrons emitted from a single-particle source in the quantum Hall regime, Phys. Rev. B 85, 075428 (2012).

[64] L.-H. Reydellet, P. Roche, D. C. Glattli, B. Etienne, and Y. Jin, Quantum Partition Noise of Photon-Created Electron-Hole Pairs, Phys. Rev. Lett. 90, 176803 (2003).

[65] V. S. Rychkov, M. L. Polianski, and M. Büttiker, Photonassisted electron-hole shot noise in multiterminal conductors, Phys. Rev. B 72, 155326 (2005).

[66] M. Vanević, Y. V. Nazarov, and W. Belzig, Elementary Events of Electron Transfer in a Voltage-Driven Quantum Point Contact, Phys. Rev. Lett. 99, 076601 (2007).

[67] M. Vanević, Y. V. Nazarov, and W. Belzig, Elementary chargetransfer processes in mesoscopic conductors, Phys. Rev. B 78, 245308 (2008).

[68] M. Albert, C. Flindt, and M. Büttiker, Accuracy of the quantum capacitor as a single-electron source, Phys. Rev. B 82, 041407(R) (2010).

[69] A. Mahé, F. D. Parmentier, E. Bocquillon, J.-M. Berroir, D. C. Glattli, T. Kontos, B. Plaçais, G. Fève, A. Cavanna, and Y. Jin,
Current correlations of an on-demand single-electron emitter, Phys. Rev. B 82, 201309(R) (2010).

[70] F. D. Parmentier, E. Bocquillon, J.-M. Berroir, D. C. Glattli, B. Plaçais, G. Fève, M. Albert, C. Flindt, and M. Büttiker, Current noise spectrum of a single-particle emitter: Theory and experiment, Phys. Rev. B 85, 165438 (2012).

[71] M. Moskalets, Noise of a single-electron emitter, Phys. Rev. B 88, 035433 (2013).

[72] M. Vanević, J. Gabelli, W. Belzig, and B. Reulet, Electron and electron-hole quasiparticle states in a driven quantum contact, Phys. Rev. B 93, 041416(R) (2016).

[73] A. Crépieux and F. Michelini, Mixed, charge and heat noises in thermoelectric nanosystems, J. Phys.: Condens. Matter 27, 015302 (2014).

[74] A. Crépieux and F. Michelini, Heat-charge mixed noise and thermoelectric efficiency fluctuations, J. Stat. Mech.: Theory Exp. (2016) 054015.

[75] P. Eyméoud and A. Crépieux, Mixed electrical-heat noise spectrum in a quantum dot, Phys. Rev. B 94, 205416 (2016).

[76] Ya. M. Blanter and M. Büttiker, Shot noise in mesoscopic conductors, Phys. Rep. 336, 1 (2000).

[77] D. Ferraro, A. Feller, A. Ghibaudo, E. Thibierge, E. Bocquillon, G. Fève, Ch. Grenier, and P. Degiovanni, Wigner function approach to single electron coherence in quantum Hall edge channels, Phys. Rev. B 88, 205303 (2013).

[78] Handbook of Mathematical Functions with Formulas, Graphs, and Mathematical Tables, edited by M. Abramowitz and I. Stegun, 10th ed., Applied Mathematics Series Vol. 55 (United States Department of Commerce, National Bureau of Standards, Washington D.C., 1972, reprinted by Dover Publications, New York). 